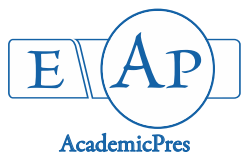

Samal SK et al. (2021)

Notulae Scientia Biologicae

Volume 13, Issue 4, Article number 11074

DOI: $10.15835 / \mathrm{nsb} 13411074$

Research Article

\title{
Diversity of butterflies (Lepidoptera: Rhopalocera) in Bhubaneswar, Odisha, India
}

\section{Sabindra K. SAMAL*, Aryjit SATAPATHY, Nivedita PATTANAIK}

\author{
B.J.B. Autonomous College, Department of Zoology, B.J.B. Nagar, Bhubaneswar - 751014, Odisha, India; \\ sabindra.kr.samal@gmail.com (*correspondingauthor); aryjit89@gmail.com;nivedita.p22@gmail.com \\ All authors contributed equally to the work
}

\begin{abstract}
The loss and fragmentation of habitat caused by rapid urbanization can have devastating effects, both at regional and global level. In this study, butterfly species diversity has been assessed in Bhubaneswar, India, as a model geographical region for understanding the biology of the local population and its dynamics. In total 107 butterfly species have been documented, with the highest number of species being recorded from the family Nymphalidae (31.77\%), followed by Lycaenidae (25.23\%), Hesperiidae (23.36\%), Pieridae $(11.21 \%)$ and Papilionidae (8.41\%). Out of these, 17 species are new reports for the city and nine species are legally protected in India under the Wildlife (Protection) Act, 1972. Sørensen's diversity index and one-way ANOVA have been used to establish the relation between species diversity and habitat. The present investigation provides baseline data for future research and conservation of species in places like the model city, which face rapid urbanization.
\end{abstract}

Keywords: Bhubaneswar; butterfly diversity; conservation; Lepidoptera; new reports; scheduled species

\section{Introduction}

Order Lepidoptera comprises butterflies and moths which belong to the class Insecta (Kunte, 2000; Kawahara and Breinholt, 2014). Butterflies occupy a vital position in the ecosystem, acting as pollinators, pollution indicators, good source of food, and have aesthetic importance (Klein et al., 2007; Syaripuddin et al., 2015). Their studies have made significant contributions towards the understanding of biogeography, behaviour, coevolution, conservation, development, ecological genetics, global warming, mimicry, population ecology, sexual selection, speciation, symbiotic associations, and systematics (Aduse-Poku et al., 2015; Kozak et al., 2015; Kronforst and Papa, 2015; Manesi et al., 2015; Wang Wei et al., 2016; van Bergen et al., 2017). Mature and immature individuals that have narrow niches often show specificity towards their host plants (Tiple et al., 2011; Salz and Fartmann, 2017; Nallu et al., 2018; Verspagen et al., 2020).

Therefore, butterflies are regarded as good indicators of the quality of microhabitat and the extent of associated anthropogenic disturbances (Ruszczyk and Silva, 1997; Kehimkar, 2016). Approximately, 18,768 species of butterflies have been recorded worldwide (Van Nieukerken et al., 2011) and recent findings suggest that India hosts 1318 species in its subcontinent, out of which 89 species belong to Papilionidae, 277 species belong to Hesperiidae, 92 species belong to Pieridae, 19 species belong to Riodinidae, 380 species belong to Lycaenidae, and 461 species belong to Nymphalidae (Cotton et al., 2015; Kehimkar, 2016). Butterfly works in 
Indian subcontinent are pioneered by Horsfield and Moore (1857). Marshall and Nicéville (1882), Colonel Bingham $(1905,1907)$ and Talbot $(1939,1947)$ studied butterflies in India and documented them in 'The Fauna of British India'.

The state Odisha is situated at latitudes $17^{\circ} 49^{\prime}-22^{\circ} 33^{\prime} \mathrm{N}$ and longitudes $81^{\circ} 27^{\prime}-87^{\circ} 57^{\prime} \mathrm{E}$ in the eastern peninsula and experiences a tropical savanna climate with an average annual rainfall of $1450 \mathrm{~mm}$ to $1600 \mathrm{~mm}$ and an average annual temperature of $24.4{ }^{\circ} \mathrm{C}$ to $32.7^{\circ} \mathrm{C}$ (India Meteorological Department, 2018: https://metnet.imd.gov.in/imdnews/ar2018.pdf, Forest \& Environmental Department, 2020: http://www.orienvis.nic.in/). Presence of distinct phytogeographical regions such as the Eastern Ghats, the Deccan Plateau and the coastal plains with tropical forest cover and deciduous forests are appropriate habitats for faunal diversity (Reddy et al., 2014). The faunistic works on butterflies of the state were pioneered by Taylor and Nicéville, where they prepared a list of butterfly species from Puri and Khurda district in 1888, followed by Crawford in 1921 from Sambalpur district. Annandale and Dover also recorded the butterfly species of Barkuda Island of Ganjam district in 1921 (Mandal and Nandi, 1983).

Though literature related to the butterfly diversity and abundance is available for the state (Mishra et al., 2010; Mohapatra et al., 2013; Priyamvada and Mohapatra, 2016; Boruah et al., 2018; Payra et al., 2019; Singh et al., 2020), the species diversity and conservation in relation to their habitats in the rapidly urbanizing city of Bhubaneswar is yet to be explored. As part of long-term conservation strategies, local population dynamics as well as species diversity become extremely important since habitat fragmentation and loss of microhabitat pose threats to population persistence (Thomas et al., 1992; Roy et al., 2010; Fernandez-Chacon et al., 2014; MacDonald et al., 2018). In the past decade, topology and climate of the city have changed significantly due to increased urbanization, decreased vegetation, and a rise in temperature owing to the interplay of biotic and abiotic factors (Swain et al., 2016; Gogoi et al., 2019). The study aims to document the butterfly species diversity in relation to their habitats such as open scrub, garden habitat, urban habitat, urban park, urban forest, fragmented forest, and cropland in the city and its outskirts. The present study documented a list of 107 butterfly species out of which 17 species are new reports for this region and nine species are enlisted under the Wildlife (Protection) Act, 1972 (WPA, 1972).

\section{Materials and Methods}

\section{Study site}

The study was conducted in Bhubaneswar and its outskirts in Khordha district of Odisha, India, covering approximately $440 \mathrm{~km}^{2}$. The study range was divided into 11 different study sites and maps indicating the same were created using ArcGIS software (version 10.3) and Google Earth (Figure 1). The detailed information about Global Positioning System (GPS) coordinates, elevation, and habitats for each study sites are provided (Table 1). The study range is predominated by seven types of habitats (Figure 2).

\section{Survey and monitoring methods}

A survey and documentation of the study sites were conducted from July 2018 to August 2020. For data collection, opportunistic survey and random sightings were used along with modified Pollard walk method (Pollard and Yates, 1992; Royer et al., 1998; Wood and Gillman, 1998; Pellet et al., 2012). All observations were carried out between $6: 30 \mathrm{hrs}$ to $11: 30 \mathrm{hrs}$ and 14:30 hrs to $17: 30 \mathrm{hrs}$ in good weathered conditions. During the study tenure, each study site was visited at least two times in each quarter of a year. Information about the coordinates and elevation of study sites were obtained by using Google's location services. Butterflies were photographed using Nikon gears (D3500 and D5300 DSLR cameras mounted with 18-55 mm and 70$300 \mathrm{~mm}$ Nikkor lenses), identified using field guides, books and the butterflies of India website (Kunte, 2000; Kehimkar, 2016; Smetacek, 2018; Butterflies of India, 2021: https://www.ifoundbutterflies.org/). The WPA, 1972 status of the butterflies were obtained from the database available at ENVIS Center on Wildlife \& 
Protected Areas (Scheduled Insect species, 2014: http://wiienvis.nic.in/). An entomological net was occasionally used and the butterflies were released unharmed to their natural habitat as soon as they were photographed. In this study none of the butterfly species were collected, euthanized or killed by any means. The host plants were identified and recorded as per the literature available (Karmakar et al., 2018; Nitin et al., 2018).

1

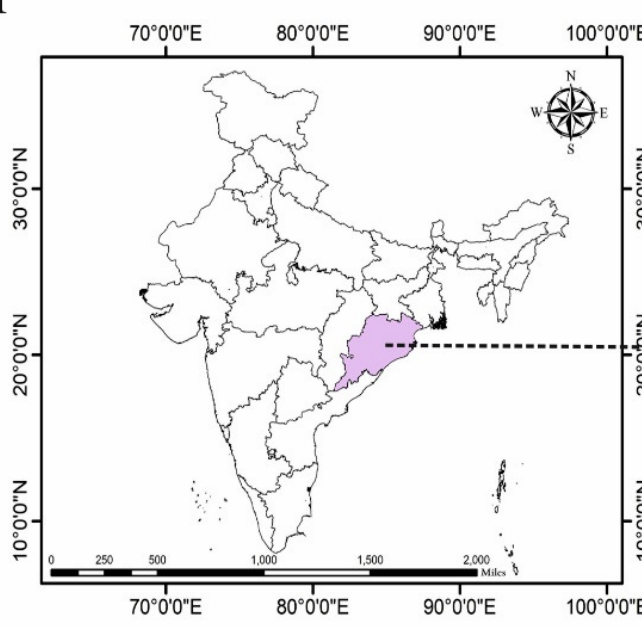

4

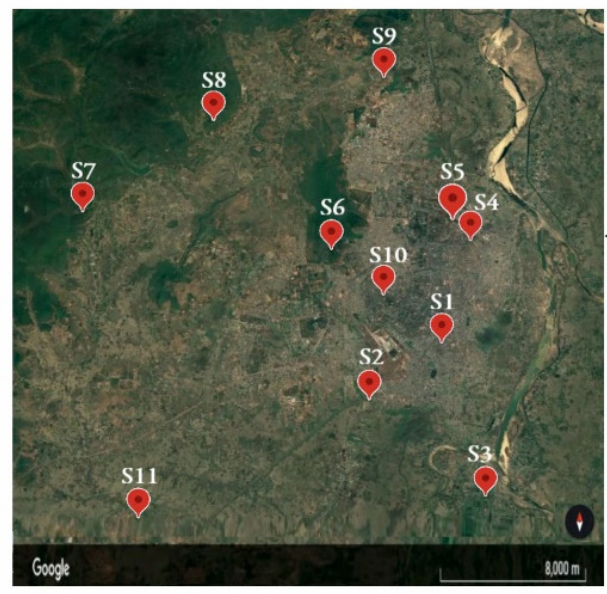

2
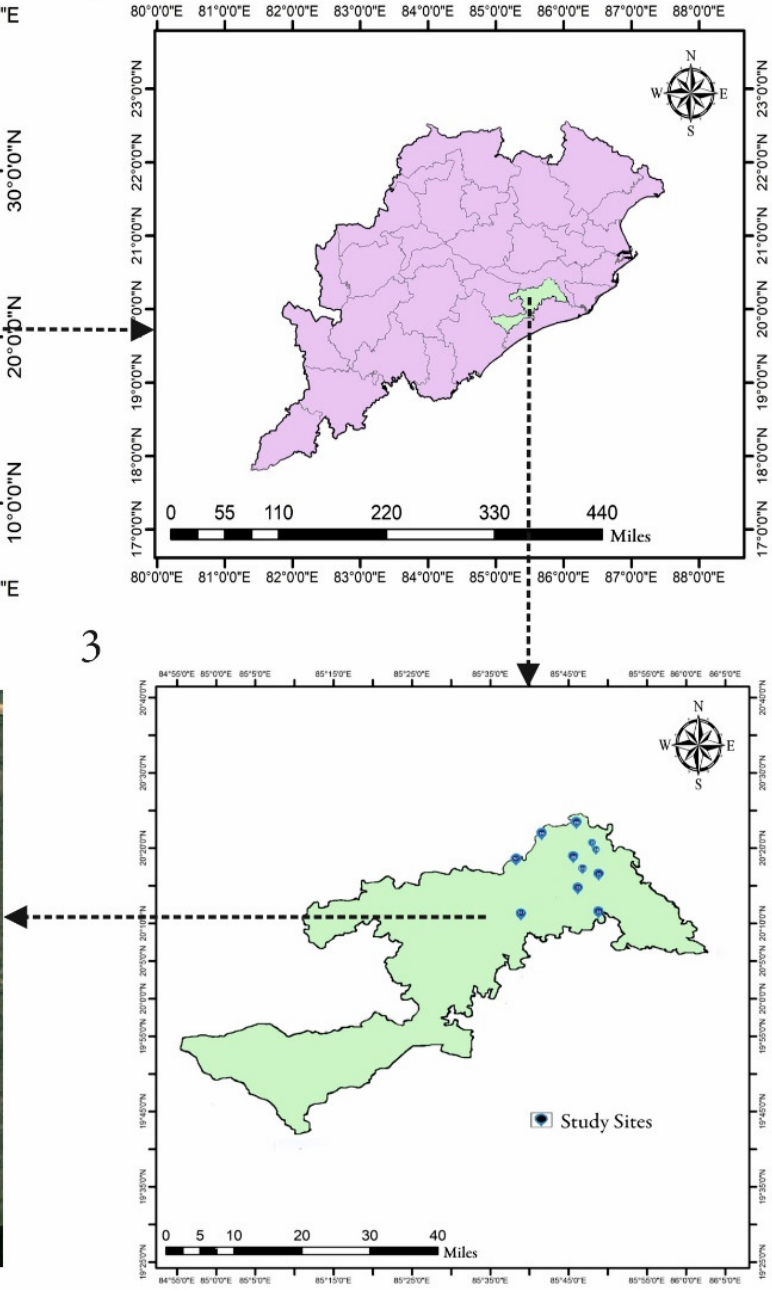

Figure 1. Maps indicating study sites: (1) Map of India indicating the state Odisha; (2) Map of the state Odisha indicating Khordha district; (3) Khordha district indicating the study sites of Bhubaneswar and its outskirts; (4) Google map indicating the topology of the study range with marked study sites S1-S11 are the study sites 
Table 1. Physiographic information about the study sites of Bhubaneswar and its outskirts

\begin{tabular}{|c|c|c|c|c|c|}
\hline $\begin{array}{l}\text { Sl. } \\
\text { no. }\end{array}$ & $\begin{array}{l}\text { Study } \\
\text { site }\end{array}$ & Name of the study site & GPS coordinates & Elevation & Habitats \\
\hline 1 & S1 & B. J. B. College Campus & $\begin{array}{l}20.2506^{\circ} \mathrm{N} \\
85.8411^{\circ} \mathrm{E}\end{array}$ & $35 \mathrm{~m} / 115 \mathrm{ft}$ & $\begin{array}{l}\text { Open scrub, Garden habitat, } \\
\text { Urban habitat, Urban park }\end{array}$ \\
\hline 2 & $\mathrm{~S} 2$ & Old Town & $\begin{array}{l}20.2388^{\circ} \mathrm{N} \\
85.8346^{\circ} \mathrm{E}\end{array}$ & $36 \mathrm{~m} / 118 \mathrm{ft}$ & $\begin{array}{l}\text { Urban habitat having roadside } \\
\text { plantation }\end{array}$ \\
\hline 3 & S3 & Dhauli Village & $\begin{array}{l}20.1972^{\circ} \mathrm{N} \\
85.8419^{\circ} \mathrm{E}\end{array}$ & $40 \mathrm{~m} / 131 \mathrm{ft}$ & $\begin{array}{c}\text { Open scrub, Urban forest with } \\
\text { water bodies }\end{array}$ \\
\hline 4 & S4 & Saheed Nagar & $\begin{array}{l}20.2910^{\circ} \mathrm{N} \\
85.8456^{\circ} \mathrm{E}\end{array}$ & $43 \mathrm{~m} / 141 \mathrm{ft}$ & $\begin{array}{c}\text { Urban habitat having roadside } \\
\text { plantation }\end{array}$ \\
\hline 5 & S5 & Vani Vihar & $\begin{array}{l}20.3040^{\circ} \mathrm{N} \\
85.8397^{\circ} \mathrm{E}\end{array}$ & $41 \mathrm{~m} / 135 \mathrm{ft}$ & $\begin{array}{c}\text { Open scrub, Urban habitat, } \\
\text { Urban forest }\end{array}$ \\
\hline 6 & S6 & Nayapalli & $\begin{array}{l}20.2997^{\circ} \mathrm{N} \\
85.8060^{\circ} \mathrm{E}\end{array}$ & $58 \mathrm{~m} / 190 \mathrm{ft}$ & $\begin{array}{c}\text { Open scrub, Garden habitat, } \\
\text { Urban forest }\end{array}$ \\
\hline 7 & S7 & $\begin{array}{l}\text { Ghangapatana and } \\
\text { Deulipatana Villages }\end{array}$ & $\begin{array}{l}20.3088^{\circ} \mathrm{N} \\
85.7308^{\circ} \mathrm{E}\end{array}$ & $46 \mathrm{~m} / 151 \mathrm{ft}$ & $\begin{array}{c}\text { Open scrub, Fragmented forest } \\
\text { having bamboo patches, } \\
\text { Cropland }\end{array}$ \\
\hline 8 & S8 & Chandaka Village & $\begin{array}{l}20.3489^{\circ} \mathrm{N} \\
85.7346^{\circ} \mathrm{E}\end{array}$ & $107 \mathrm{~m} / 351 \mathrm{ft}$ & $\begin{array}{l}\text { Fragmented forest having } \\
\text { bamboo patches, Cropland }\end{array}$ \\
\hline 9 & S9 & Raghunathpur & $\begin{array}{l}20.3958^{\circ} \mathrm{N} \\
85.8260^{\circ} \mathrm{E}\end{array}$ & $23 \mathrm{~m} / 75 \mathrm{ft}$ & $\begin{array}{c}\text { Open scrub, Fragmented forest, } \\
\text { Cropland }\end{array}$ \\
\hline 10 & S10 & Surya Nagar & $\begin{array}{c}20.2698^{\circ} \mathrm{N} \\
85.8131^{\circ} \mathrm{E}\end{array}$ & $40 \mathrm{~m} / 131 \mathrm{ft}$ & $\begin{array}{l}\text { Urban habitat having roadside } \\
\text { plantation }\end{array}$ \\
\hline 11 & S11 & Barunei & $\begin{array}{l}20.1611^{\circ} \mathrm{N} \\
85.6461^{\circ} \mathrm{E}\end{array}$ & $130 \mathrm{~m} / 427 \mathrm{ft}$ & $\begin{array}{l}\text { Open scrub, Urban forest, } \\
\text { Fragmented forest }\end{array}$ \\
\hline
\end{tabular}
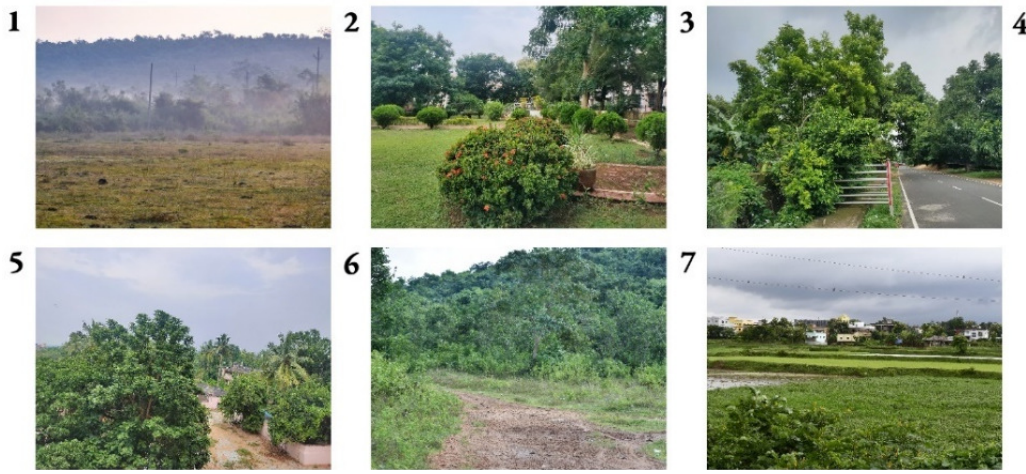

Figure 2. Types of habitats in the study range: (1) Open scrub; (2) Garden habitat; (3) Urban habitat; (4) Urban park; (5) Urban forest; (6) Fragmented forest; (7) Cropland

\section{Statistical analysis}

Sørensen's method was applied to find the similarity between number of species in different study sites and one-way Analysis of Variance (ANOVA) was used to analyse the dependence of relative distribution of species on habitat.

\section{Sørensen's similarity index $(\beta)$}

The Sørensen index is an indicator of similarity between two communities based on the number of common species shared by them. $\beta$ can be defined as:

$$
\beta=2 c /(s 1+s 2)
$$


where, $s 1=$ total number of species recorded in the first community, $s 2=$ number of species recorded in the second community, and $c=$ number of species common to both communities.

The value of $\beta$ lies between 0 and 1 , wherein as the value approaches 0 , the species overlap between the communities decrease and as the value moves towards 1 , both the communities start having a greater number of shared species (Christopher, 2020). All the calculations were performed using Microsoft Excel (version 2019).

\section{One-way ANOVA}

It is used to determine the differences in the means of three or more independent groups. One-way ANOVA has been used in this study to establish a substantial relation between the variation in habitat and the species diversity by considering a null hypothesis $\left(\mathrm{H}_{0}\right)$ that the mean of family wise species diversity of each site is the same. The alternative hypothesis $\left(\mathrm{H}_{1}\right)$ is that at least one of the means is different. A significance level $(\alpha)$ of 0.05 implies a $5 \%$ risk of concluding that a difference exists when there is no actual difference. The variance ratio, $\mathrm{F}$ statistic ( $\mathrm{F}$ stat), probability value ( $\mathrm{P}$-value), and critical value of $\mathrm{F}$ distribution ( $\mathrm{F}$ crit) in the ANOVA table serve as the base to conclude the analysis. If $\mathrm{P}<0.05$ and the value of $\mathrm{F}$ stat is more than $\mathrm{F}$ crit, then the null hypothesis is rejected whereas if $\mathrm{P}>0.05$ and $\mathrm{F}$ stat is less than $\mathrm{F}$ crit, then the null hypothesis is accepted (Kim, 2017). In this study, one-way ANOVA was calculated using GraphPad Prism Software (version 5.0) and Microsoft Excel (version 2019).

\section{Results}

A total of 107 species of butterflies, belonging to 76 genera and five families (Papilionidae, Hesperiidae, Pieridae, Lycaenidae, and Nymphalidae) were recorded in this study whereas no butterflies from the families Hedylidae and Riodinidae were encountered during this study (Table 2). Information about host plants of the recorded butterfly species were enlisted (Table 3). Butterfly hierarchy was represented showing the number of species observed from each family (Figure 3). Photographs of each species categorised into their respective families were represented (Figure 4-8). Out of the total number of species recorded, nine species are legally protected in India under WPA, 1972, and 17 species are new reports for the city (Table 3). The highest number of species have been observed in Nymphalidae (34 species, 31.77\%), followed by Lycaenidae (27 species, 25.23\%), Hesperiidae (25 species, 23.36\%), Pieridae (12 species, 11.21\%), and Papilionidae (nine species, 8.41\%) as illustrated (Figure 9A). The species to genus ratio (S/G) determines distribution of species among genera, and is calculated to be 3.000, 1.316, 1.500, 1.125, and 1.545 for Papilionidae, Hesperiidae, Pieridae, Lycaenidae, and Nymphalidae respectively (Figure 9B, Table 4). The family wise species distribution in different study sites showed that the highest number is observed in S8 (88 species) followed by $S 7$ ( 77 species), S9 (74 species), S5 (63 species), S6 (48 species), S11 ( 43 species), S10 (42 species), S1 (36 species), S3 (34 species), S2 (31 species), and the lowest number is observed in S4 (27 species) as illustrated (Figure 9C). The distribution range of species number is found to be more scattered for Hesperiidae, Lycaenidae, and Nymphalidae as compared to Papilionidae and Pieridae (Figure 9D). According to the Sørensen's similarity index, a maximum value of 0.896 is observed between study sites $S 1$ and $S 2$ whereas a minimum value of 0.470 is observed between study sites $S 4$ and $S 8$ (Table 5). One-way ANOVA data shows that $\mathrm{F}$ stat value is 13.92, value of $\mathrm{F}$ crit is 2.55 , and $\mathrm{P}<0.05$ for butterfly species in different families within the study range. Between the butterfly species among the study sites the result shows that $\mathrm{F}$ stat value is 2.34 , value of $\mathrm{F}$ crit is 2.05 , and $\mathrm{P}<0.05$. In both the cases $\mathrm{P}<0.05$ and the value of $\mathrm{F}$ stat is greater than the $\mathrm{F}$ crit value (Table 6). 
Samal SK et al. (2021). Not Sci Biol 13(4):11074

Table 2. List of butterflies with detailed information recorded during this study

\begin{tabular}{|c|c|c|c|c|c|c|}
\hline $\begin{array}{l}\text { Sl. } \\
\text { no. }\end{array}$ & $\begin{array}{l}\text { Family/Sub } \\
\text { Family }\end{array}$ & Common name & Scientific name & Authority & $\begin{array}{l}\text { Adult wing span } \\
\text { (in } \mathrm{mm} \text { ) }\end{array}$ & $\begin{array}{l}\text { WPA, } \\
1972\end{array}$ \\
\hline \multicolumn{7}{|c|}{ Family Papilionidae: The swallow tails } \\
\hline 1 & \multirow{9}{*}{$\begin{array}{l}\text { Sub Family: } \\
\text { Papilioninae }\end{array}$} & Blue mormon & Papilio polymnestor & Cramer, 1775 & $120-150$ & \\
\hline 2 & & $\begin{array}{c}\text { Common banded } \\
\text { peacock }\end{array}$ & Papilio crino & Fabricius, 1793 & $80-100$ & \\
\hline 3 & & Common jay & Graphium doson & $\begin{array}{c}\text { (C. \& R. Felder, } \\
1864)\end{array}$ & $70-80$ & \\
\hline 4 & & Common mime & Papilio clytia & Linnaeus, 1758 & $90-100$ & Sch I \\
\hline 5 & & Common mormon & Papilio polytes & Linnaeus, 1758 & $90-100$ & \\
\hline 6 & & Common rose & $\begin{array}{c}\text { Pachliopta } \\
\text { aristolochiae }\end{array}$ & (Fabricius, 1775) & $80-110$ & \\
\hline 7 & & Crimson rose & Pachliopta hector & (Linnaeus, 1758) & $90-120$ & Sch I \\
\hline 8 & & Lime & Papilio demoleus & Linnaeus, 1758 & $80-100$ & \\
\hline 9 & & Tailed jay & $\begin{array}{c}\text { Graphium } \\
\text { agamemnon }\end{array}$ & (Linnaeus, 1758) & $85-100$ & \\
\hline \multicolumn{7}{|c|}{ Family Hedylidae: The Moth-butterflies. No butterflies were recorded during this study } \\
\hline \multicolumn{7}{|c|}{ Family Hesperiidae: The skippers } \\
\hline 10 & Sub Family: & $\begin{array}{c}\text { Common banded } \\
\text { awl }\end{array}$ & Hasora chromus & (Cramer, $[1780])$ & $45-50$ & \\
\hline 11 & Coentadinae & Plain banded awl^ & Hasora vitta & (Butler, 1870) & $45-55$ & Sch IV \\
\hline 12 & \multirow{18}{*}{$\begin{array}{l}\text { Sub Family: } \\
\text { Hesperiinae }\end{array}$} & Bush hopper & Ampittia dioscorides & (Fabricius, 1793) & $22-28$ & \\
\hline 13 & & Chestnut bob & Iambrix salsala & (Moore, $[1866])$ & $26-30$ & \\
\hline 14 & & Common grass dart & Taractrocera maevius & (Fabricius, 1793) & $22-28$ & \\
\hline 15 & & Common redeye & Matapa aria & (Moore, [1866]) & $40-55$ & \\
\hline 16 & & Continental swift ${ }^{\wedge}$ & Parnara ganga & (Evans, 1937) & $30-32$ & \\
\hline 17 & & Dark palm dart & Telicota aneilla & (Moore, 1878) & $33-36$ & \\
\hline 18 & & Grass demon & Udaspes folus & (Cramer, $[1775])$ & $40-48$ & \\
\hline 19 & & Indian palm bob & Suastus gremius & (Fabricius, 1798) & $32-45$ & \\
\hline 20 & & $\begin{array}{c}\text { Large branded } \\
\text { swift }^{\wedge}\end{array}$ & Pelopidas subochracea & (Moore, 1878) & $38-42$ & \\
\hline 21 & & Lesser rice swift ${ }^{\wedge}$ & Borbo bevani & (Moore, 1878) & $32-36$ & \\
\hline 22 & & $\begin{array}{l}\text { Little branded } \\
\text { swift^ }\end{array}$ & Pelopidas agna & (Moore, [1866]) & $30-38$ & \\
\hline 23 & & Pale palm dart ${ }^{\wedge}$ & Telicota colon & (Fabricius, 1775) & $30-36$ & \\
\hline 24 & & Plain palm dart ${ }^{\wedge}$ & Cephrenes acalle & (Höpffer, 1874) & 45 & \\
\hline 25 & & Purple redeye $^{\wedge}$ & Matapa purpurascens & $\begin{array}{c}\text { Elwes \& Edwards, } \\
1897\end{array}$ & $48-54$ & \\
\hline 26 & & Rice swift & Borbo cinnara & (Wallace, 1866) & $30-36$ & \\
\hline 27 & & Small branded swift & Pelopidas mathias & (Fabricius, 1798) & $32-38$ & \\
\hline 28 & & Tree flitter & Hyarotis adrastus & (Stoll, $[1780]$ ) & $38-48$ & Sch IV \\
\hline 29 & & Wax dart & Cupitha purreea & (Moore, 1877) & $28-33$ & \\
\hline 30 & \multirow{3}{*}{$\begin{array}{l}\text { Sub Family: } \\
\text { Pyrginae }\end{array}$} & Common small flat & Sarangesa dasahara & (Moore, [1866]) & $26-35$ & \\
\hline 31 & & Golden angle & Caprona ransonnetti & (R. Felder, 1868) & $35-45$ & \\
\hline 32 & & Indian skipper & Spialia galba & (Fabricius, 1793) & $22-27$ & \\
\hline
\end{tabular}


Samal SK et al. (2021). Not Sci Biol 13(4):11074

\begin{tabular}{|c|c|c|c|c|c|c|}
\hline 33 & & Tricolour pied flat ${ }^{\wedge}$ & Coladenia indrani & (Moore, [1866]) & $40-46$ & \\
\hline 34 & & Water snow flat ${ }^{\wedge}$ & Tagiades litigiosa & Möschler, 1878 & $37-44$ & \\
\hline \multicolumn{7}{|c|}{$\begin{array}{c}\text { Family Pieridae: The whites and yellows } \\
\end{array}$} \\
\hline 35 & \multirow{6}{*}{$\begin{array}{l}\text { Sub Family: } \\
\text { Coliadinae }\end{array}$} & 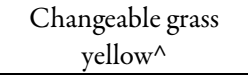 & Eurema simulatrix & $\begin{array}{c}\text { (Staudinger, } \\
1891) \\
\end{array}$ & $40-50$ & \\
\hline 36 & & Common emigrant & Catopsilia pomona & (Fabricius, 1775) & $55-80$ & \\
\hline 37 & & $\begin{array}{l}\text { Common grass } \\
\text { yellow }\end{array}$ & Eurema hecabe & (Linnaeus, 1758) & $40-50$ & \\
\hline 38 & & Mottled emigrant & Catopsilia pyranthe & (Linnaeus, 1758) & $50-70$ & \\
\hline 39 & & $\begin{array}{c}\text { One-spot grass } \\
\text { yellow }\end{array}$ & Eurema andersonii & (Moore, 1886) & $38-45$ & \\
\hline 40 & & $\begin{array}{c}\text { Three-spot grass } \\
\text { yellow }\end{array}$ & Eurema blanda & (Boisduval, 1836) & $40-45$ & \\
\hline 41 & \multirow{6}{*}{$\begin{array}{l}\text { Sub Family: } \\
\text { Pierinae }\end{array}$} & $\begin{array}{c}\text { Black veined } \\
\text { albatross }\end{array}$ & Appias olferna & Swinhoe, 1890 & $55-65$ & \\
\hline 42 & & Common gull & Cepora nerissa & (Fabricius, 1775) & $40-65$ & \\
\hline 43 & & Common jezebel & Delias eucharis & (Drury, 1773) & $66-83$ & \\
\hline 44 & & Common wanderer & Pareronia hippia & (Fabricius, 1787) & $65-80$ & \\
\hline 45 & & Psyche & Leptosia nina & (Fabricius, 1793) & $35-50$ & \\
\hline 46 & & Yellow orange-tip & Ixias pyrene & (Linnaeus, 1764) & $50-70$ & \\
\hline \multicolumn{7}{|c|}{ Family Riodinidae: The Metal-markers. No butterflies were recorded during this study } \\
\hline \multicolumn{7}{|c|}{ Family Lycaenidae: The gossamer-winged butterflies } \\
\hline 47 & $\begin{array}{l}\text { Sub Family: } \\
\text { Curetinae }\end{array}$ & Indian sunbeam & Curetis thetis & (Drury, $[1773])$ & $40-48$ & \\
\hline 48 & \multirow{18}{*}{$\begin{array}{l}\text { Sub Family: } \\
\text { Polyommatinae }\end{array}$} & $\begin{array}{c}\text { Black-spotted grass } \\
\text { jewel }^{\wedge}\end{array}$ & Freyeria putli & (Kollar, [1844]) & $12-18$ & \\
\hline 49 & & Common cerulean & Jamides celeno & (Cramer, $[1775])$ & $27-40$ & \\
\hline 50 & & $\begin{array}{l}\text { Common hedge } \\
\text { blue }\end{array}$ & Acytolepis puspa & $\begin{array}{c}\text { (Horsfield, } \\
[1828]) \\
\end{array}$ & $28-35$ & \\
\hline 51 & & Common lineblue & Prosotas nora & (Felder, 1860) & $18-25$ & \\
\hline 52 & & Common pierrot & Castalius rosimon & (Fabricius, 1775) & $24-34$ & \\
\hline 53 & & Dark cerulean & Jamides bochus & (Stoll, [1782]) & $25-34$ & \\
\hline 54 & & Dark grass blue & Zizeeria karsandra & (Moore, 1865) & $18-24$ & \\
\hline 55 & & Forget-me-not & Catochrysops strabo & (Fabricius, 1793) & $25-35$ & \\
\hline 56 & & Gram blue & Euchrysops cnejus & (Fabricius, 1798) & $25-33$ & Sch II \\
\hline 57 & & Lesser grass blue & Zizina otis & (Fabricius, 1787) & $19-26$ & \\
\hline 58 & & Lime blue & Chilades lajus & (Stoll, $[1780])$ & $26-30$ & \\
\hline 59 & & Little tiger pierrot $^{\wedge}$ & Tarucus balkanica & (Freyer, 1844) & $21-24$ & \\
\hline 60 & & Pale grass blue & Pseudozizeeria maha & (Kollar, [1844]) & $26-30$ & \\
\hline 61 & & Pea blue & Lampides boeticus & (Linnaeus, 1767) & $24-36$ & Sch II \\
\hline 62 & & Plains cupid & Luthrodes pandava & (Horsfield, 1829) & $25-33$ & \\
\hline 63 & & Pointed ciliate blue & Anthene lycaenina & (Felder, 1868) & $24-29$ & Sch II \\
\hline 64 & & Quaker & $\begin{array}{c}\text { Neopithecops } \\
\text { zalmora }\end{array}$ & (Butler, [1870]) & $16-30$ & \\
\hline 65 & & Silver forget-me-not & $\begin{array}{l}\text { Catochrysops } \\
\text { panormus }\end{array}$ & (Felder, 1860) & $25-35$ & \\
\hline
\end{tabular}


Samal SK et al. (2021). Not Sci Biol 13(4):11074

\begin{tabular}{|c|c|c|c|c|c|c|}
\hline 66 & & Striped pierrot & Tarucus nara & (Kollar, 1848) & $16-28$ & \\
\hline 67 & & Tiny grass blue & Zizula hylax & (Fabricius, 1775) & $16-24$ & \\
\hline 68 & \multirow{6}{*}{$\begin{array}{l}\text { Sub Family: } \\
\text { Theclinae }\end{array}$} & Common silverline & Spindasis vulcanus & (Fabricius, 1775) & $26-34$ & \\
\hline 69 & & Falcate oakblue ${ }^{\wedge}$ & Mahathala ameria & (Hewitson, 1862) & $38-42$ & Sch II \\
\hline 70 & & Monkey puzzle & Rathinda amor & (Fabricius, 1775) & $26-28$ & \\
\hline 71 & & Purple leaf blue & Amblypodia anita & Hewitson, 1862 & $45-52$ & \\
\hline 72 & & Slate flash & Rapala manea & (Hewitson, 1863) & $30-33$ & \\
\hline 73 & & Yamfly & Loxura atymnus & (Stoll, 1780) & $36-40$ & \\
\hline \multicolumn{7}{|c|}{ Family Nymphalidae: The brush-footed butterflies } \\
\hline 74 & \multirow{2}{*}{$\begin{array}{l}\text { Sub Family: } \\
\text { Biblidinae }\end{array}$} & Angled castor & Ariadne ariadne & (Moore, 1884) & $45-60$ & \\
\hline 75 & & Common castor & Ariadne merione & (Cramer, [1777]) & $45-60$ & \\
\hline 76 & $\begin{array}{l}\text { Sub Family: } \\
\text { Charaxinae }\end{array}$ & Common nawab & Polyura athamas & (Drury, 1773) & $60-75$ & \\
\hline 77 & \multirow{5}{*}{$\begin{array}{l}\text { Sub Family: } \\
\text { Danainae }\end{array}$} & Common crow & Euploea core & (Cramer, $[1780])$ & $85-95$ & \\
\hline 78 & & Blue tiger & Tirumala limniace & (Cramer, $[1775])$ & $90-100$ & \\
\hline 79 & & Glassy tiger & Parantica aglea & (Stoll, [1782]) & $70-85$ & \\
\hline 80 & & Plain tiger & Danaus chrysippus & (Linnaeus, 1758) & $70-80$ & \\
\hline 81 & & Striped tiger & Danaus genutia & (Cramer, [1779]) & $72-100$ & \\
\hline 82 & \multirow{2}{*}{$\begin{array}{l}\text { Sub Family: } \\
\text { Heliconiinae }\end{array}$} & Common leopard & Phalanta phalantha & Drury, 1773 & $50-60$ & \\
\hline 83 & & Tawny coster & Acraea terpsicore & (Linnaeus, 1758) & $50-65$ & \\
\hline 84 & \multirow{6}{*}{$\begin{array}{l}\text { Sub Family: } \\
\text { Limenitidinae }\end{array}$} & Baronet & Symphaedra nais & (Forster, 1771) & $60-70$ & \\
\hline 85 & & $\begin{array}{l}\text { Chestnut-streaked } \\
\text { sailer }\end{array}$ & Neptis jumbah & Moore, [1858] & $60-70$ & \\
\hline 86 & & Commander & Moduza procris & (Cramer, [1777]) & $60-75$ & \\
\hline 87 & & Common baron & Euthalia aconthea & (Cramer, [1777]) & $55-80$ & \\
\hline 88 & & Common lascar & Pantoporia hordonia & (Stoll, $[1790])$ & $45-50$ & \\
\hline 89 & & Common sailer & Neptis hylas & (Linnaeus, 1758) & $50-60$ & \\
\hline 90 & $\begin{array}{l}\text { Sub Family: } \\
\text { Morphinae }\end{array}$ & Common duffer $^{\wedge}$ & Discophora sondaica & Boisduval, 1836 & $80-90$ & \\
\hline 91 & \multirow{8}{*}{$\begin{array}{l}\text { Sub Family: } \\
\text { Nymphalinae }\end{array}$} & Blue pansy & Junonia orithya & (Linnaeus, 1758) & $45-60$ & \\
\hline 92 & & Chocolate pansy & Junonia iphita & (Cramer, [1779]) & $55-80$ & \\
\hline 93 & & Danaid eggfly & Hypolimnas misippus & (Linnaeus, 1764) & $70-85$ & Sch I \\
\hline 94 & & Great eggfly & Hypolimnas bolina & (Linnaeus, 1758) & $70-110$ & \\
\hline 95 & & Grey pansy & Junonia atlites & (Linnaeus, 1763) & $55-60$ & \\
\hline 96 & & Lemon pansy & Junonia lemonias & (Linnaeus, 1758) & $40-60$ & \\
\hline 97 & & Painted lady^d & Vanessa cardui & (Linnaeus, 1758) & $55-70$ & \\
\hline 98 & & Peacock pansy & Junonia almana & (Linnaeus, 1758) & $60-65$ & \\
\hline 99 & \multirow{4}{*}{$\begin{array}{l}\text { Sub Family: } \\
\text { Satyrinae }\end{array}$} & Bamboo tree brown & Lethe europa & (Fabricius, 1787) & $65-75$ & \\
\hline 100 & & $\begin{array}{c}\text { Common } \\
\text { bushbrown }\end{array}$ & Mycalesis perseus & (Fabricius, 1775) & $38-55$ & \\
\hline 101 & & $\begin{array}{c}\text { Common evening } \\
\text { brown }\end{array}$ & Melanitis leda & (Linnaeus, 1758) & $60-80$ & \\
\hline 102 & & Common four-ring & Ypthima huebneri & Kirby, 1871 & $30-40$ & \\
\hline
\end{tabular}


Samal SK et al. (2021). Not Sci Biol 13(4):11074

\begin{tabular}{|c|c|c|c|c|}
\hline 103 & Common palmfly & $\begin{array}{c}\text { Elymnias } \\
\text { hypermnestra }\end{array}$ & (Linnaeus, 1763) & $60-80$ \\
\hline 104 & $\begin{array}{c}\text { Common three- } \\
\text { ring }^{\wedge}\end{array}$ & Ypthima asterope & (Klug, 1832) & $30-37$ \\
\hline 105 & $\begin{array}{c}\text { Dark- branded } \\
\text { bushbrown }\end{array}$ & Mycalesis mineus & (Linnaeus, 1858) & $40-50$ \\
\hline 106 & $\begin{array}{c}\text { Dark evening } \\
\text { brown }\end{array}$ & Melanitis phedima & (Cramer, $[1780])$ & $60-70$ \\
\hline 107 & White four-ring & Ypthima ceylonica & Hewitson, [1865] & $30-35$ \\
\hline
\end{tabular}

Species marked with $(\wedge)$ reported for the first time from Bhubaneswar. Species marked with $\left({ }^{\mathrm{d}}\right)$ is long range migratory species. mm, millimeter; Sch, Scheduled species; WPA, 1972, The Wildlife (Protection) Act, 1972.

Table 3. Host plant list of the recorded butterfly species

\begin{tabular}{|c|c|c|c|c|}
\hline \multirow{2}{*}{$\begin{array}{l}\text { Sl. } \\
\text { no. }\end{array}$} & \multirow{2}{*}{ Butterfly species } & \multicolumn{3}{|c|}{ Host plant } \\
\hline & & Family & Common name & Scientific name \\
\hline 1 & Papilio polymnestor & $\begin{array}{l}\text { i) Rubiaceae } \\
\text { ii) Rutaceae }\end{array}$ & $\begin{array}{l}\text { i) Jungle geranium } \\
\text { ii) Pomelo }\end{array}$ & $\begin{array}{l}\text { i) Ixora coccinea } \\
\text { ii) Citrus maxima }\end{array}$ \\
\hline 2 & Papilio crino & Rutaceae & $\begin{array}{c}\text { a) Lemon } \\
\text { b) East Indian satinwood }\end{array}$ & $\begin{array}{l}\text { a) Citrus limon } \\
\text { b) Chloroxylon swietenia }\end{array}$ \\
\hline 3 & Graphium doson & $\begin{array}{l}\text { i) Annonaceae } \\
\text { ii) Magnoliaceae }\end{array}$ & $\begin{array}{l}\text { i) a) Pond apple } \\
\text { b) Hoom } \\
\text { c) False Ashoka/Buddha tree } \\
\text { ii) a) Southern Magnolia/Him } \\
\text { champa } \\
\text { b) Champak/Champa }\end{array}$ & $\begin{array}{l}\text { i) a) Annona glabra } \\
\text { b) Miliusa tomentosa } \\
\text { c) Polyalthia longifolia } \\
\text { ii) a) Magnolia grandiflora } \\
\text { b) Michelia champaca }\end{array}$ \\
\hline 4 & Papilio clytia & Lauraceae & $\begin{array}{l}\text { a) Camphor tree/Camphorwood } \\
\text { b) Indian bay leaf }\end{array}$ & $\begin{array}{l}\text { a) Cinnamomum camphora } \\
\text { b) Cinnamomum tamala }\end{array}$ \\
\hline 5 & Papilio polytes & Rutaceae & $\begin{array}{l}\text { a) Bael/Wood apple } \\
\text { b) Curry leaf tree } \\
\text { c) Citron/Galgal } \\
\text { d) Persian lime }\end{array}$ & $\begin{array}{l}\text { a) Aegle marmelos } \\
\text { b) Murraya koenigii } \\
\text { c) Citrus medica } \\
\text { d) Citrus latifolia }\end{array}$ \\
\hline 6 & Pachliopta aristolochiae & Aristolochiaceae & $\begin{array}{l}\text { a) Indian birthwort } \\
\text { b) Calico flower/Pipe vine } \\
\text { c) Chakrani }\end{array}$ & $\begin{array}{l}\text { a) Aristolochia indica } \\
\text { b) Aristolochia littoralis } \\
\text { c) Thottea siliquosa }\end{array}$ \\
\hline 7 & Pachliopta hector & Aristolochiaceae & $\begin{array}{l}\text { a) Indian birthwort } \\
\text { b) Calico flower/Pipe vine }\end{array}$ & $\begin{array}{l}\text { a) Aristolochia indica } \\
\text { b) Aristolochia littoralis }\end{array}$ \\
\hline 8 & Papilio demoleus & $\begin{array}{l}\text { i) Fabaceae } \\
\text { ii) Rhamnaceae } \\
\text { iii) Rutaceae }\end{array}$ & $\begin{array}{l}\text { i) Babchi/Kushtanashini } \\
\text { ii) Jujube/Indian plum } \\
\text { iii) a) Bael/Wood apple } \\
\text { b) East Indian Satinwood } \\
\text { c) Citron/Galgal } \\
\text { d) Elephant-apple/Kaith } \\
\text { e) Curry leaf tree }\end{array}$ & $\begin{array}{l}\text { i) Psoralea corylifolia } \\
\text { ii) Ziziphus mauritiana } \\
\text { iii) a) Aegle marmelos } \\
\text { b) Chloroxylon swietania } \\
\text { c) Citrus medica } \\
\text { d) Limonia acidissima } \\
\text { e) Muraya koenigii }\end{array}$ \\
\hline 9 & Graphium agamemnon & $\begin{array}{l}\text { i) Annonaceae } \\
\text { ii) Magnoliaceae }\end{array}$ & $\begin{array}{l}\text { i) a) Pond apple } \\
\text { b) Hoom } \\
\text { c) False Ashoka/Buddha tree } \\
\text { d) Corky debbar tree } \\
\text { ii) Champak/Champa }\end{array}$ & $\begin{array}{l}\text { i) a) Annona glabera } \\
\text { b) Miliusa tomentosa } \\
\text { c) Polyalthia longifolia } \\
\text { d) Polyalthia suberosa } \\
\text { ii) Michelia champaca }\end{array}$ \\
\hline 10 & Hasora chromus & Fabaceae & Karanja/Indian beech & Millettia pinnata \\
\hline 11 & Hasora vitta & Fabaceae & $\begin{array}{l}\text { a) Arge-leaf pongam } \\
\text { b) Ardhga/Swardhang }\end{array}$ & $\begin{array}{l}\text { a) Millettia extensa } \\
\text { b) Endosamara racemose }\end{array}$ \\
\hline 12 & Ampittia dioscorides & Poaceae & Asian rice & Oryza sativa \\
\hline 13 & Iambrix salsala & Poaceae & $\begin{array}{l}\text { a) Indian thorny bamboo } \\
\text { b) Para grass/Buffalo grass }\end{array}$ & $\begin{array}{l}\text { a) Bambusa arundinacea } \\
\text { b) Brachiaria mutica }\end{array}$ \\
\hline 14 & Taractrocera maevius & Poaceae & Asian rice & Oryza sativa \\
\hline
\end{tabular}


Samal SK et al. (2021). Not Sci Biol 13(4):11074

\begin{tabular}{|c|c|c|c|c|}
\hline 15 & Matapa aria & Poaceae & Indian thorny bamboo & Bambusa arundinaceae \\
\hline 16 & Parnara ganga & Poaceae & Asian rice & Oryza sativa \\
\hline 17 & Telicota aneilla & Poaceae & $\begin{array}{l}\text { a) Sugarcanes } \\
\text { b) Common bamboo }\end{array}$ & $\begin{array}{l}\text { a) Saccharum sp. } \\
\text { b) Bambusa vulgaris }\end{array}$ \\
\hline 18 & Udaspes folus & $\begin{array}{l}\text { i) Costaceae } \\
\text { ii) Zingiberaceae }\end{array}$ & $\begin{array}{l}\text { i) Crepe ginger } \\
\text { ii) Black Turmeric }\end{array}$ & $\begin{array}{l}\text { i) Costus speciosus } \\
\text { ii) Curcuma caesia }\end{array}$ \\
\hline 19 & Suastus gremius & Arecaceae & $\begin{array}{l}\text { a) Queen palm } \\
\text { b) Coconut tree } \\
\text { c) Golden cane palm } \\
\text { d) Silver date palm/Indian date }\end{array}$ & $\begin{array}{l}\text { a) Arecastrum } \\
\text { romanziffianum } \\
\text { b) Cocos nucifera } \\
\text { c) Dypsis lutescens } \\
\text { d) Phoenix sylvestris }\end{array}$ \\
\hline 20 & Pelopidas subochracea & Poaceae & Carpet grass & Axonopus compressus \\
\hline 21 & Borbo bevani & Poaceae & $\begin{array}{l}\text { a) Sugarcanes } \\
\text { b) Cogon grass }\end{array}$ & $\begin{array}{l}\text { a) Saccharum sp. } \\
\text { b) Imperata cylindrica }\end{array}$ \\
\hline 22 & Pelopidas agna & Poaceae & $\begin{array}{l}\text { a) Carpet grass } \\
\text { b) Fountain grass }\end{array}$ & $\begin{array}{l}\text { a) Axonopus compressus } \\
\text { b) Pennisetum sp. }\end{array}$ \\
\hline 23 & Telicota colon & Poaceae & Sugarcane & Saccharum officinarum \\
\hline 24 & Cephrenes acalle & Arecaceae & $\begin{array}{l}\text { a) Coconut tree } \\
\text { b) Palmyra palm }\end{array}$ & $\begin{array}{l}\text { a) Cocos nucifera } \\
\text { b) Borassus flabellifer }\end{array}$ \\
\hline 25 & Matapa purpurascens & Asteraceae & Siam weed/Tibra gandha & Chromolaena odorata \\
\hline 26 & Borbo cinnara & Poaceae & $\begin{array}{l}\text { a) Para grass/Buffalo grass } \\
\text { b) Asian rice } \\
\text { c) Johnson grass } \\
\text { d) Jowar }\end{array}$ & $\begin{array}{l}\text { a) Brachiaria mutica } \\
\text { b) Oryza sativa } \\
\text { c) Sorghum halepense } \\
\text { d) Sorghum nitidum }\end{array}$ \\
\hline 27 & Pelopidas mathias & Poaceae & $\begin{array}{l}\text { a) Sugarcane } \\
\text { b) Asian rice }\end{array}$ & $\begin{array}{l}\text { a) Saccharum officinarum } \\
\text { b) Oryza sativa }\end{array}$ \\
\hline 28 & Hyarotis adrastus & Arecaceae & Rattan palms & Calamus sp. \\
\hline 29 & Cupitha purreea & Combretaceae & $\begin{array}{l}\text { a) Rangoon creeper } \\
\text { b) Bahada }\end{array}$ & $\begin{array}{l}\text { a) Combretum indicum } \\
\text { b) Terminalia bellirica }\end{array}$ \\
\hline 30 & Sarangesa dasahara & Acanthaceae & $\begin{array}{l}\text { a) Violet asystasia } \\
\text { b) Spiny lepidagathis }\end{array}$ & $\begin{array}{l}\text { a) Asystasia dalzelliana } \\
\text { b) Lepidagathis cuspidata }\end{array}$ \\
\hline 31 & Caprona ransonnetti & Malvaceae & Indian screw tree & Helicteres isora \\
\hline 32 & Spialiagalba & $\begin{array}{l}\text { i) Malvaceae } \\
\text { ii) Sterculiaceae }\end{array}$ & $\begin{array}{l}\text { i) a) Common wireweed } \\
\text { b) Chocolateweed } \\
\text { ii) Sleepy morning }\end{array}$ & $\begin{array}{l}\text { i) a) Sida acuta } \\
\text { b) Melochia corchorifolia } \\
\text { ii) Waltheria indica }\end{array}$ \\
\hline 33 & Coladenia indrani & $\begin{array}{l}\text { i) Fabaceae } \\
\text { ii) Malvaceae }\end{array}$ & $\begin{array}{l}\text { a) Bidi leaf tree } \\
\text { ii) Diamond burbark }\end{array}$ & $\begin{array}{l}\text { i) Bauhinia racemosa } \\
\text { ii) Triumfetta rhomboidea }\end{array}$ \\
\hline 34 & Tagiades litigiosa & $\begin{array}{l}\text { i) Dioscoreaceae } \\
\text { ii) Dipterocarpaceae }\end{array}$ & $\begin{array}{l}\text { i) Bana alu/Pita alu } \\
\text { ii) Taloora lac tree }\end{array}$ & $\begin{array}{l}\text { i) Dioscorea wallichii } \\
\text { ii) Shorea roxburghii }\end{array}$ \\
\hline 35 & Eurema simulatrix & Fabaceae & Golden shower/Indian laburnum & Cassia fistula \\
\hline 36 & Catopsilia pomona & $\begin{array}{l}\text { i) Apocynaceae } \\
\text { ii) Fabaceae }\end{array}$ & $\begin{array}{l}\text { i) Conkerberry/Bush plum } \\
\text { ii) a) Bidi leaf tree } \\
\text { b) Flame-of-the-forest/Palash } \\
\text { c) Golden Shower/Indian laburnum } \\
\text { d) Vegetable Hummingbird }\end{array}$ & $\begin{array}{l}\text { i) Carissa spinarum } \\
\text { ii) a) Bauhinia racemosa } \\
\text { b) Butea monosperma } \\
\text { c) Cassia fistula } \\
\text { d) Sesbania grandiflora }\end{array}$ \\
\hline 37 & Eurema hecabe & Fabaceae & $\begin{array}{l}\text { a) Rain tree/Monkey pod } \\
\text { b) Peacock flower/Red bird of } \\
\text { paradise } \\
\text { c) Red powder puff } \\
\text { d) Golden shower/Indian laburnum }\end{array}$ & $\begin{array}{l}\text { a) Albizia saman } \\
\text { b) Caesalpinia pulcherrima } \\
\text { c) Calliandra haematocephala } \\
\text { d) Cassia fistula }\end{array}$ \\
\hline 38 & Catopsilia pyranthe & $\begin{array}{l}\text { i) Apocynaceae } \\
\text { ii) Fabaceae }\end{array}$ & $\begin{array}{l}\text { i) Conkerberry/Bush plum } \\
\text { ii) Golden shower/Indian laburnum }\end{array}$ & $\begin{array}{l}\text { i) Carissa spinarum } \\
\text { ii) Cassia fistula }\end{array}$ \\
\hline 39 & Eurema andersonii & Rhamnaceae & Toothed-leaf red creeper & Ventilago denticulata \\
\hline 40 & Eurema blanda & Fabaceae & Purple orchid tree & Bauhinia purpurea \\
\hline 41 & Appias olferna & Capparaceae & Fringed spider flower & Cleome rutidosperma \\
\hline
\end{tabular}


Samal SK et al. (2021). Not Sci Biol 13(4):11074

\begin{tabular}{|c|c|c|c|c|}
\hline 42 & Cepora nerissa & Capparaceae & $\begin{array}{l}\text { a) Caper shrubs/Caperbushes } \\
\text { b) Ceylon caper }\end{array}$ & $\begin{array}{l}\text { a) Capparis sp. } \\
\text { b) Capparis zeylanica }\end{array}$ \\
\hline 43 & Delias eucharis & $\begin{array}{l}\text { i) Loranthaceae } \\
\text { ii) Meliaceae }\end{array}$ & $\begin{array}{l}\text { i) Honey suckle mistletoe } \\
\text { ii) Neem }\end{array}$ & $\begin{array}{l}\text { i) Dendrophthoe falcata } \\
\text { ii) Azadirachta indica }\end{array}$ \\
\hline 44 & Pareronia hippia & Capparaceae & $\begin{array}{l}\text { a) Caper shrubs/Caperbushes } \\
\text { b) Ceylon caper }\end{array}$ & $\begin{array}{l}\text { a) Capparis sp. } \\
\text { b) Capparis zeylanica }\end{array}$ \\
\hline 45 & Leptosia nina & Capparaceae & $\begin{array}{l}\text { a) Fringed spiderflower/Purple } \\
\text { cleome } \\
\text { b) Asian spiderflower/Tick weed } \\
\text { c) Caper shrubs/Caper bushes }\end{array}$ & $\begin{array}{l}\text { a) Cleome rutidosperma } \\
\text { b) Cleome viscosa } \\
\text { c) Capparis sp. }\end{array}$ \\
\hline 46 & Ixias pyrene & Capparaceae & $\begin{array}{l}\text { a) Spreading caper } \\
\text { b) Wild caper bush }\end{array}$ & $\begin{array}{l}\text { a) Capparis divaricata } \\
\text { b) Capparis sepiaria }\end{array}$ \\
\hline 47 & Curetis thetis & Fabaceae & $\begin{array}{l}\text { a) Jequirity bean/Rosary pea } \\
\text { b) Flame-of-the-forest/Palash } \\
\text { c) Karanja/Indian beech }\end{array}$ & $\begin{array}{l}\text { a) Abrus precatorius } \\
\text { b) Butea monosperma } \\
\text { d) Milletia pinnata }\end{array}$ \\
\hline 48 & Freyeria putli & $\begin{array}{l}\text { i) Asteraceae } \\
\text { ii) Boraginaceae } \\
\text { iii) Fabaceae }\end{array}$ & $\begin{array}{l}\text { i) Tridax daisy } \\
\text { ii) Indian heliotrope } \\
\text { iii) Narrow-leafed indigo }\end{array}$ & $\begin{array}{l}\text { i) Tridax procumbens } \\
\text { ii) Heliotropium indicum } \\
\text { iii) Indigofera linifolia }\end{array}$ \\
\hline 49 & Jamides celeno & $\begin{array}{l}\text { i) Fabaceae } \\
\text { ii) Zingiberaceae }\end{array}$ & $\begin{array}{l}\text { i) a) Ashoka tree } \\
\text { b) Mung bean/Green gram } \\
\text { ii) Green/True cardamom }\end{array}$ & $\begin{array}{l}\text { i) a) Saraca asoca } \\
\text { b) Vigna radiata } \\
\text { ii) Elettaria cardamomum }\end{array}$ \\
\hline 50 & Acytolepis puspa & $\begin{array}{l}\text { i) Fabaceae } \\
\text { ii) Phyllanthaceae } \\
\text { iii) Sapindaceae }\end{array}$ & $\begin{array}{l}\text { i) Mexican lilac } \\
\text { ii) Spinous kino tree } \\
\text { iii) Kurpa }\end{array}$ & $\begin{array}{l}\text { i) Gliricidia sepium } \\
\text { ii) Bridelia retusa } \\
\text { iii) Lepisanthes tetraphylla }\end{array}$ \\
\hline 51 & Prosotas nora & $\begin{array}{l}\text { i) Euphorbiaceae } \\
\text { ii) Mimosaceae } \\
\text { iii) Sapindaceae }\end{array}$ & $\begin{array}{c}\text { i) Kamala/Kumkum tree } \\
\text { ii) Twisted acacia } \\
\text { iii) Indian allophylus/Tit berry }\end{array}$ & $\begin{array}{l}\text { i) Mallotus philippensis } \\
\text { ii) Acacia torta } \\
\text { iii) Allophylus cobbe }\end{array}$ \\
\hline 52 & Castalius rosimon & Rhamnaceae & $\begin{array}{l}\text { a) Jujube/Red date } \\
\text { b) Jhar beri }\end{array}$ & $\begin{array}{l}\text { a) Ziziphus zuzuba } \\
\text { b) Ziziphus nummularia }\end{array}$ \\
\hline 53 & Jamides bochus & Fabaceae & $\begin{array}{l}\text { a) Ashoka tree } \\
\text { b) Burma ironwood }\end{array}$ & $\begin{array}{l}\text { a) Saraca asoca } \\
\text { b) Xylia xylocarpa }\end{array}$ \\
\hline 54 & Zizeeria karsandra & $\begin{array}{l}\text { i) Amaranthaceae } \\
\text { ii) Molluginaceae } \\
\text { iii) Polygonaceae } \\
\text { iv) Zygophyllaceae }\end{array}$ & $\begin{array}{l}\text { i) Spiny amaranthus } \\
\text { ii) Lotus sweetjuice } \\
\text { iii) Common knotweed } \\
\text { iv) Bindii }\end{array}$ & $\begin{array}{l}\text { i) Amaranthus spinosus } \\
\text { ii) Glinus lotoides } \\
\text { iii) Polygonum plebeium } \\
\text { iv) Tribulus terrestris }\end{array}$ \\
\hline 55 & Catochrysops strabo & $\begin{array}{l}\text { i) Fabaceae } \\
\text { ii) Sapindaceae }\end{array}$ & $\begin{array}{l}\text { i) a) Asian tick trefoil } \\
\text { b) Karanja/Indian beech } \\
\text { c) Common tephrosia } \\
\text { ii) Indian Allophylus/Tit berry }\end{array}$ & $\begin{array}{l}\text { i) a) Desmodium } \\
\text { heterocarpum } \\
\text { b) Millettia pinnata } \\
\text { c) Tephrosia purpurea } \\
\text { ii) Allophylus cobbe }\end{array}$ \\
\hline 56 & Euchrysops cnejus & Fabaceae & $\begin{array}{l}\text { a) Flame-of-the-forest/Palash } \\
\text { b) Sword bean }\end{array}$ & $\begin{array}{l}\text { a) Butea monosperma } \\
\text { b) Canavalia gladiata }\end{array}$ \\
\hline 57 & Zizina otis & Fabaceae & $\begin{array}{l}\text { a) Asian tick trefoil } \\
\text { b) Chinchani/Kansevari } \\
\text { c) Three-leaf indigo }\end{array}$ & $\begin{array}{l}\text { a) Desmodium heterophyllum } \\
\text { b) Sesbania bispinosa } \\
\text { c) Indigofera trifoliata }\end{array}$ \\
\hline 58 & Chilades lajus & Rutaceae & $\begin{array}{l}\text { a) Sweet lemon } \\
\text { b) Lemon } \\
\text { c) Pomelo } \\
\text { d) Kamini/Orange jasmine }\end{array}$ & $\begin{array}{l}\text { a) Citrus limetta } \\
\text { b) Citrus limon } \\
\text { c) Citrus maxima } \\
\text { d) Murraya paniculata }\end{array}$ \\
\hline 59 & Tarucus balkanica & Rhamnaceae & $\begin{array}{l}\text { a) Jujuba/Red date } \\
\text { b) Jhar beri }\end{array}$ & $\begin{array}{l}\text { a) Ziziphus jujuba } \\
\text { b) Ziziphus nummularia }\end{array}$ \\
\hline 60 & Pseudozizeeria maha & Oxalidaceae & Creeping woodsorrel & Oxalis corniculata \\
\hline 61 & Lampides boeticus & Fabaceae & $\begin{array}{l}\text { a) Flame-of-the-forest/Palash } \\
\text { b) Garden pea }\end{array}$ & $\begin{array}{l}\text { a) Butea monosperma } \\
\text { b) Pisum sativum }\end{array}$ \\
\hline
\end{tabular}


Samal SK et al. (2021). Not Sci Biol 13(4):11074

\begin{tabular}{|c|c|c|c|c|}
\hline 62 & Luthrodes pandava & $\begin{array}{l}\text { i) Cycadaceae } \\
\text { ii) Fabaceae }\end{array}$ & $\begin{array}{l}\text { i) a) Queen sago } \\
\text { b) Nepal cycas/Thaljimura } \\
\text { ii) a) Malu creeper/Adda leaf } \\
\text { b) Orchid tree } \\
\text { c) Sword bean } \\
\text { d) Ashoka tree }\end{array}$ & $\begin{array}{l}\text { i) a) Cycas circinalis } \\
\text { b) Cycas pectinata } \\
\text { c) Cycas revoluta } \\
\text { ii) a) Bauhinia vahlii } \\
\text { b) Bauhinia variegata } \\
\text { c) Canavalia gladiata } \\
\text { d) Saraca asoca }\end{array}$ \\
\hline 63 & Anthene lycaenina & $\begin{array}{l}\text { i) Anacardiaceae } \\
\text { ii) Euphorbiaceae } \\
\text { iii) Fabaceae }\end{array}$ & $\begin{array}{c}\text { i) Chironji/Charoli } \\
\text { ii) Putranjiva } \\
\text { iii) a) Rosewood/Indian palisandre } \\
\text { b) Shikakai }\end{array}$ & $\begin{array}{l}\text { i) Buchanania lanzan } \\
\text { ii) Drypetes roxburghii } \\
\text { iii) a) Dalbergia latifolia } \\
\text { b) Acacia concinna }\end{array}$ \\
\hline 64 & Neopithecops zalmora & Rutaceae & Orange berry/Gin berry & Glycosmis pentaphylla \\
\hline 65 & $\begin{array}{l}\text { Catochrysops } \\
\text { panormus }\end{array}$ & Fabaceae & Large leaf fleminga & Flemingia macrophylla \\
\hline 66 & Tarucus nara & Rhamnaceae & $\begin{array}{l}\text { a) Jujuba/Red date } \\
\text { b) Jhar beri }\end{array}$ & $\begin{array}{l}\text { a) Ziziphus jujuba } \\
\text { b) Ziziphus nummularia }\end{array}$ \\
\hline 67 & Zizula hylax & $\begin{array}{l}\text { i) Acanthaceae } \\
\text { ii) Verbenaceae }\end{array}$ & $\begin{array}{l}\text { i) a) Temple plant/Marsh barbel } \\
\text { b) Desert petunia } \\
\text { ii) Raimuniya }\end{array}$ & $\begin{array}{l}\text { i) a) Hygrophila auriculata } \\
\text { b) Ruellia simplex } \\
\text { ii) Lantana camara }\end{array}$ \\
\hline 68 & Spindasis vulcanus & $\begin{array}{l}\text { i) Apocynaceae } \\
\text { ii) Dioscoreaceae } \\
\text { iii) Fabaceae } \\
\text { iv) Rhamnaceae }\end{array}$ & $\begin{array}{l}\text { i) Bengal currant/Karanda } \\
\text { ii) Bana alu/Pita alu } \\
\text { iii) Golden shower/Indian laburnum } \\
\text { iv) Jujube/Red date }\end{array}$ & $\begin{array}{l}\text { i) Carissa carandas } \\
\text { ii) Dioscorea wallichii } \\
\text { iii) Cassia fistula } \\
\text { iv) Ziziphus zuzuba }\end{array}$ \\
\hline 69 & Mahathala ameria & Euphorbiaceae & Climbing liana & Mallotus repandus \\
\hline 70 & Rathinda amor & $\begin{array}{l}\text { i) Anacardiaceae } \\
\text { ii) Lecythidaceae } \\
\text { iii) Rubiaceae } \\
\text { iv) Sapindaceae }\end{array}$ & $\begin{array}{l}\text { i) Mango } \\
\text { ii) Wild guava } \\
\text { iii) Jungle geranium } \\
\text { iv) Litchi }\end{array}$ & $\begin{array}{l}\text { i) Mangifera indica } \\
\text { ii) Careya arborea } \\
\text { iii) Lxora coccinea } \\
\text { iv) Litchi chinensis }\end{array}$ \\
\hline 71 & Amblypodia anita & Olacaceae & South Asian olax & Olax imbricata \\
\hline 72 & Rapala manea & $\begin{array}{l}\text { i) Combretaceae } \\
\text { ii) Lamiaceae } \\
\text { iii) Verbenaceae }\end{array}$ & $\begin{array}{l}\text { i) Rangoon creeper } \\
\text { ii) Bhat/Hill glory flower } \\
\text { iii) Raimuniya }\end{array}$ & $\begin{array}{l}\text { i) Combretum indicum } \\
\text { ii) Clerodendrum } \\
\text { infortunatum } \\
\text { iii) Lantana camara }\end{array}$ \\
\hline 73 & Loxura atymnus & $\begin{array}{l}\text { i) Dioscoreaceae } \\
\text { ii) Smilacaceae }\end{array}$ & $\begin{array}{l}\text { i) Five-leaf yam } \\
\text { ii) Kumarika }\end{array}$ & $\begin{array}{l}\text { i) Dioscorea pentaphylla } \\
\text { ii) Smilax zeylanica }\end{array}$ \\
\hline 74 & Ariadne ariadne & Euphorbiaceae & $\begin{array}{l}\text { a) Castor bean/Castor } \\
\text { b) Indian stinging nettle }\end{array}$ & $\begin{array}{l}\text { a) Ricinus communis } \\
\text { b) Tragia involucrata }\end{array}$ \\
\hline 75 & Ariadne merione & Euphorbiaceae & $\begin{array}{l}\text { a) Castor bean/Castor } \\
\text { b) Indian stinging nettle }\end{array}$ & $\begin{array}{l}\text { a) Ricinus communis } \\
\text { b) Tragia involucrata }\end{array}$ \\
\hline 76 & Polyura athamas & Fabaceae & $\begin{array}{l}\text { a) Twisted acacia } \\
\text { b) Royal poinciana/Gulmohar } \\
\text { c) Woman's tongue tree }\end{array}$ & $\begin{array}{l}\text { a) Acacia torta } \\
\text { b) Delonix regia } \\
\text { c) Albizia lebbeck }\end{array}$ \\
\hline 77 & Euploea core & $\begin{array}{l}\text { i) Apocynaceae } \\
\text { ii) Moraceae } \\
\text { iii) Sapotaceae }\end{array}$ & $\begin{array}{c}\text { i) a) Desert rose } \\
\text { b) Bengal currant/Karanda } \\
\text { c) Oleander } \\
\text { ii) a) Indian rock fig/Rock peepal } \\
\text { b) Indian banyan } \\
\text { c) Indian fig tree/Gular } \\
\text { iii) Spanish cherry }\end{array}$ & $\begin{array}{l}\text { i) a) Adenium obesum } \\
\text { b) Carissa carandas } \\
\text { c) Nerium oleander } \\
\text { ii) a) Ficus arnottiana } \\
\text { b) Ficus benghalensis } \\
\text { c) Ficus racemosa } \\
\text { iii) Mimusops elengi }\end{array}$ \\
\hline 78 & Tirumala limniace & Apocynaceae & $\begin{array}{l}\text { a) Apple of sodom/Arak } \\
\text { b) Tropical milkweed/Bloodflower } \\
\text { c) Bread flower }\end{array}$ & $\begin{array}{l}\text { a) Calotropis procera } \\
\text { b) Asclepias curassavica } \\
\text { c) Vallaris solanacea }\end{array}$ \\
\hline 79 & Parantica aglea & Apocynaceae & $\begin{array}{l}\text { a) Crown flower } \\
\text { b) Bulbous ceropegia }\end{array}$ & $\begin{array}{l}\text { a) Calotropis gigantea } \\
\text { b) Ceropegia bulbosa }\end{array}$ \\
\hline
\end{tabular}


Samal SK et al. (2021). Not Sci Biol 13(4):11074

\begin{tabular}{|c|c|c|c|c|}
\hline 80 & Danaus chrysippus & Apocynaceae & $\begin{array}{l}\text { a) Crown flower } \\
\text { b) Tropical milkweed/Bloodflower } \\
\text { c) Balloon plant }\end{array}$ & $\begin{array}{l}\text { a) Calotropis gigantea } \\
\text { b) Asclepias curassavica } \\
\text { c) Gomphocarpus } \\
\text { physocarpus }\end{array}$ \\
\hline 81 & Danaus genutia & Apocynaceae & Tropical milkweed/Bloodflower & Asclepias curassavica \\
\hline 82 & Phalanta phalantha & Salicaceae & $\begin{array}{l}\text { a) Governor's plum/Batoko plum } \\
\text { b) Indian coffee plum } \\
\text { c) Mountain sweet thorn }\end{array}$ & $\begin{array}{l}\text { a) Flacourtia indica } \\
\text { b) Flacourtia jangomas } \\
\text { c) Flacourtia montana }\end{array}$ \\
\hline 83 & Acraea terpsicore & $\begin{array}{l}\text { i) Passifloraceae } \\
\text { ii) Violaceae }\end{array}$ & $\begin{array}{l}\text { i) a) Stinking passionflower } \\
\text { b) White buttercup } \\
\text { ii) Spade flower }\end{array}$ & $\begin{array}{l}\text { i) a) Passiflora foetida } \\
\text { b) Turnera subulata } \\
\text { ii) Hybanthus enneaspermus }\end{array}$ \\
\hline 84 & Symphaedra nais & $\begin{array}{l}\text { i) Ebenaceae } \\
\text { ii) Malvaceae }\end{array}$ & $\begin{array}{l}\text { i) Coromandel ebony/Tendu } \\
\text { ii) Phalsa }\end{array}$ & $\begin{array}{l}\text { i) Diospyros melanoxylon } \\
\text { ii) Grewia asiatica }\end{array}$ \\
\hline 85 & Neptis jumbah & Fabaceae & $\begin{array}{l}\text { a) Golden shower/Indian laburnum } \\
\text { b) Indian rosewood }\end{array}$ & $\begin{array}{l}\text { a) Cassia fistula } \\
\text { b) Dalbergia latifolia }\end{array}$ \\
\hline 86 & Moduza procris & $\begin{array}{l}\text { i) Capparaceae } \\
\text { ii) Rubiaceae }\end{array}$ & $\begin{array}{l}\text { i) Indian cadaba } \\
\text { ii) a) Ashanti blood/Red flag bush } \\
\text { b) Dhobi tree/Mussaenda } \\
\text { c) Burflower-tree/Kadamb }\end{array}$ & $\begin{array}{l}\text { i) Cadaba fruticosa } \\
\text { ii) a) Mussaenda erythrophylla } \\
\text { b) Mussaenda frondosa } \\
\text { c) Neolamarckia cadamba }\end{array}$ \\
\hline 87 & Euthalia aconthea & Anacardiaceae & $\begin{array}{l}\text { a) Cashew } \\
\text { b) Mango } \\
\end{array}$ & $\begin{array}{l}\text { a) Anacardium occidentale } \\
\text { b) Mangifera indica }\end{array}$ \\
\hline 88 & Pantoporia hordonia & Fabaceae & Kali siris & Albizia odoratissima \\
\hline 89 & Neptis hylas & $\begin{array}{l}\text { i) Fabaceae } \\
\text { ii) Malvaceae } \\
\text { iii) Salicaceae }\end{array}$ & $\begin{array}{l}\text { i) Butterfly pea } \\
\text { ii) Cotton tree } \\
\text { iii) Indian willow }\end{array}$ & $\begin{array}{l}\text { i) Centrosema pubescens } \\
\text { ii) Bombax ceiba } \\
\text { iii) Salix tetrasperma }\end{array}$ \\
\hline 90 & Discophora sondaica & Poaceae & Bamboo & Bambusa sp. \\
\hline 91 & Junonia orithya & $\begin{array}{l}\text { i) Acanthaceae } \\
\text { ii) Moraceae }\end{array}$ & $\begin{array}{l}\text { i) a) Philippine violet } \\
\text { b) Water willow } \\
\text { c) Roundleaf kariyat } \\
\text { ii) White mulberry }\end{array}$ & $\begin{array}{l}\text { i) a) Barleria cristata } \\
\text { b) Justicia procumbens } \\
\text { c) Andrographis serphylifloia } \\
\text { ii) Morus alba }\end{array}$ \\
\hline 92 & Junonia iphita & Acanthaceae & $\begin{array}{c}\text { a) Philippine violet } \\
\text { b) Marsh barbel/Kokilaksha }\end{array}$ & $\begin{array}{c}\text { a) Barleria cristata } \\
\text { b) Hygrophila auriculata }\end{array}$ \\
\hline 93 & Hypolimnas misippus & $\begin{array}{l}\text { i) Convolvulaceae } \\
\text { ii) Malvaceae }\end{array}$ & $\begin{array}{l}\text { i) Pink morning glory } \\
\text { ii) China rose }\end{array}$ & $\begin{array}{l}\text { i) Ipomoea carnea } \\
\text { ii) Hibiscus rosa-sinensis }\end{array}$ \\
\hline 94 & Hypolimnas bolina & Urticaceae & Hen's nettle & Laportea interrupta \\
\hline 95 & Junonia atlites & Acanthaceae & $\begin{array}{c}\text { a) Philippine violet } \\
\text { b) Marsh barbel/Kokilaksha }\end{array}$ & $\begin{array}{c}\text { a) Barleria cristata } \\
\text { b) Hygrophila auriculata }\end{array}$ \\
\hline 96 & Junonia lemonias & Acanthaceae & $\begin{array}{l}\text { a) Philippine violet } \\
\text { b) Water willow } \\
\text { c) Roundleaf kariyat }\end{array}$ & $\begin{array}{c}\text { a) Barleria cristata } \\
\text { b) Justicia procumbens } \\
\text { c) Andrographis serphylifloia }\end{array}$ \\
\hline 97 & Vanessa cardui & $\begin{array}{l}\text { i) Asteraceae } \\
\text { ii) Fabaceae } \\
\text { iii) Papaveraceae }\end{array}$ & $\begin{array}{l}\text { i) Coatbuttons/Tridax daisy } \\
\text { ii) Two-leaf zornia } \\
\text { iii) Mexican prickly poppy }\end{array}$ & $\begin{array}{l}\text { i) Tridax procumbens } \\
\text { ii) Zornia diphylla } \\
\text { iii) Argemome mexicana }\end{array}$ \\
\hline 98 & Junonia almana & Acanthaceae & $\begin{array}{c}\text { a) Philippine violet } \\
\text { b) Marsh barbel/Kokilaksha }\end{array}$ & $\begin{array}{l}\text { a) Barleria cristata } \\
\text { b) Hygrophila auriculata }\end{array}$ \\
\hline 99 & Lethe europa & Poaceae & Bamboo & Bambusa sp. \\
\hline 100 & Mycalesis perseus & Poaceae & $\begin{array}{l}\text { a) Running mountain grass } \\
\text { b) Barnyard grass/Cockspur grass }\end{array}$ & $\begin{array}{l}\text { a) Oplismenus compositus } \\
\text { b) Echinochloa sp. }\end{array}$ \\
\hline 101 & Melanitis leda & Poaceae & $\begin{array}{l}\text { a) Maize/Corn } \\
\text { b) Indian thorny bamboo } \\
\text { c) Indian goosegrass }\end{array}$ & $\begin{array}{l}\text { a) Zea mays } \\
\text { b) Bambusa arundinacea } \\
\text { c) Eleusine indica }\end{array}$ \\
\hline 102 & Ypthima huebneri & Poaceae & Indian goosegrass & Eleusine indica \\
\hline 103 & Elymnias hypermnestra & Arecaceae & $\begin{array}{l}\text { a) Areca palm } \\
\text { b) Golden cane palm } \\
\text { c) Silver date palm/Indian date } \\
\text { d) Macarthur palm }\end{array}$ & $\begin{array}{c}\text { a) Areca catechu } \\
\text { b) Dypsis lutescens } \\
\text { c) Phoenix sylvestris } \\
\text { d) Ptychosperma macarthurii }\end{array}$ \\
\hline
\end{tabular}




\begin{tabular}{|c|c|c|c|c|}
\hline 104 & Ypthima asterope & Poaceae & Bermuda grass/Duba ghasa & Cynodon sp. \\
\hline 105 & Mycalesis mineus & Poaceae & Corn grass & Setaria barbata \\
\hline 106 & Melanitis phedima & Poaceae & $\begin{array}{c}\text { a) Running mountain grass } \\
\text { b) Palmgrass/Knotroot }\end{array}$ & $\begin{array}{c}\text { a) Oplismenus compositus } \\
\text { b) Setaria palmifolia }\end{array}$ \\
\hline 107 & Ypthima ceylonica & Poaceae & Corn grass & Setaria barbata \\
\hline
\end{tabular}

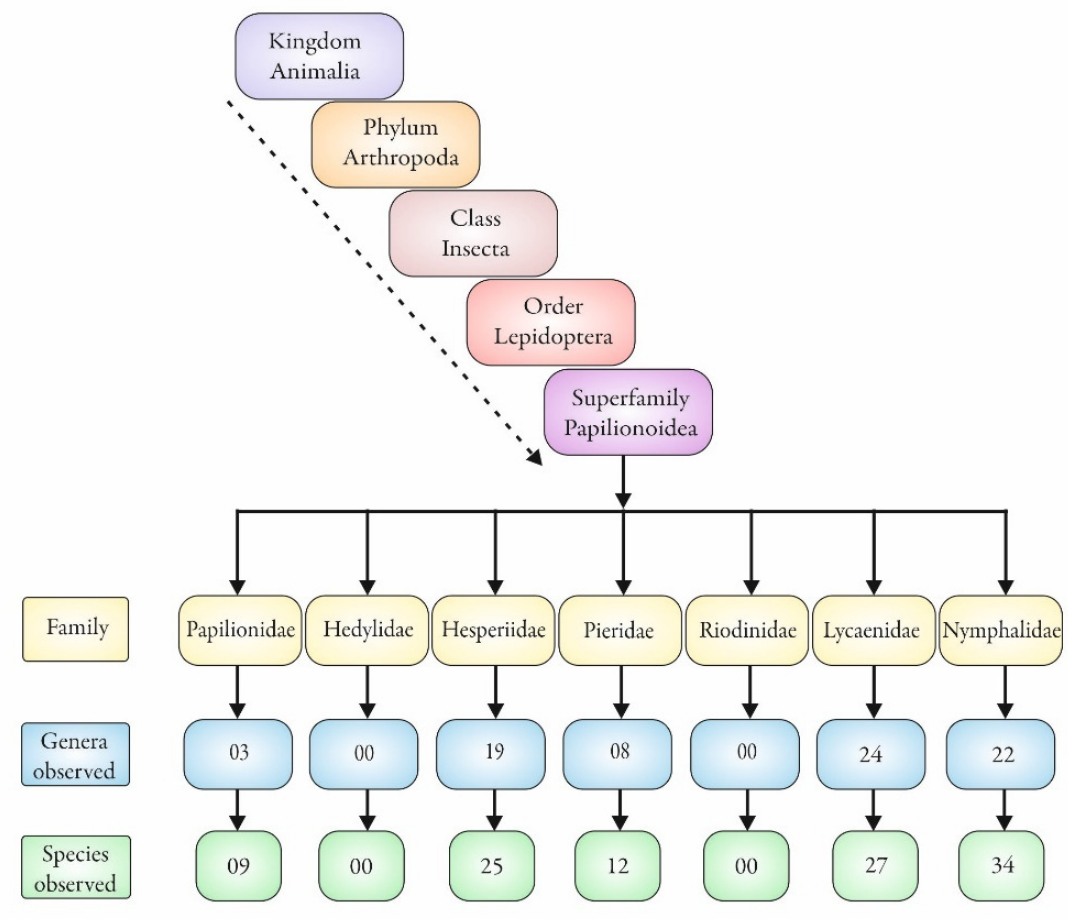

Figure 3. Hierarchy of butterfly indicating the number of species observed in this study Adapted from Van Nieukerken et al. (2011)
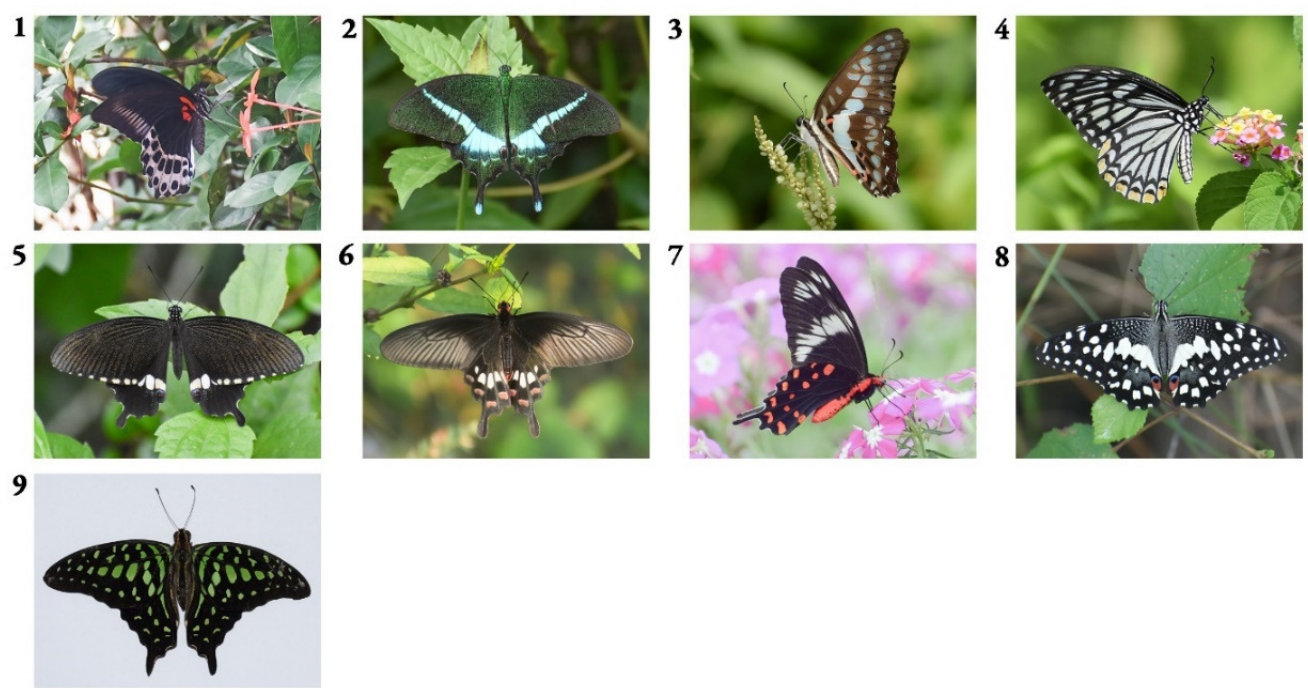

Figure 4. Photographs of butterfly species belonging to the family Papilionidae recorded in this study: (1) Papilio polymnestor; (2) Papilio crino; (3) Graphium doson; (4) Papilio clytia; (5) Papilio polytes; (6) Pachliopta aristolochiae; (7) Pachliopta hector; (8) Papilio demoleus; (9) Graphium agamemnon 
1
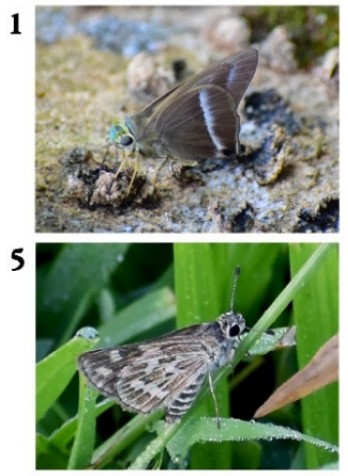

9

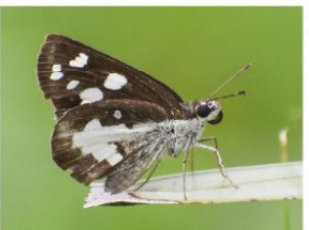

13

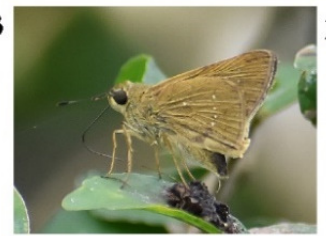

17

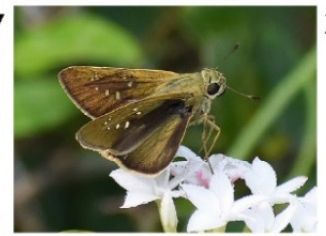

21

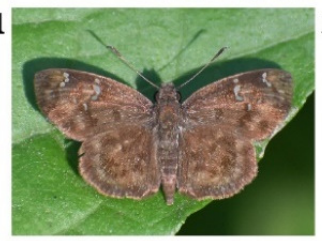

25

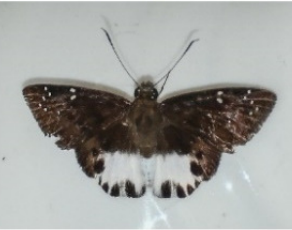

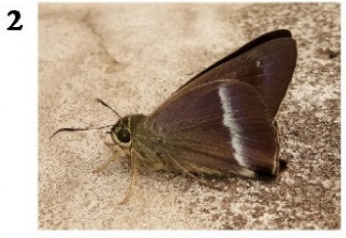

6
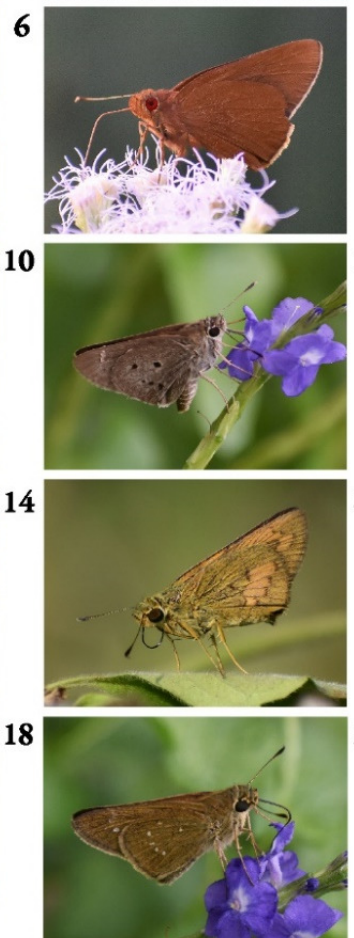

22

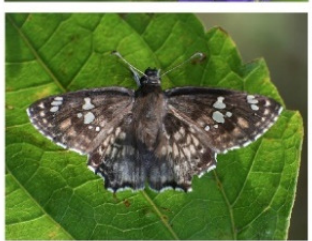

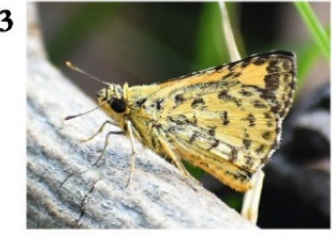

7
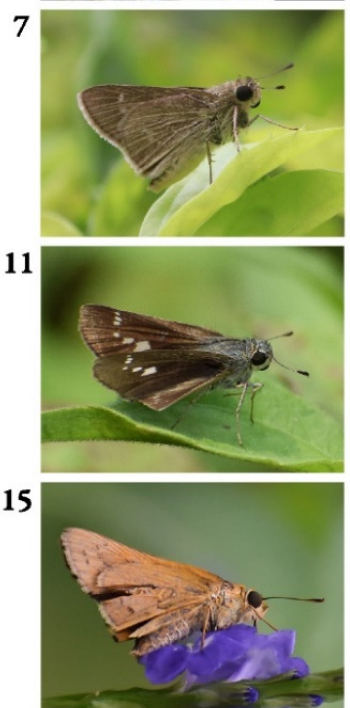

19

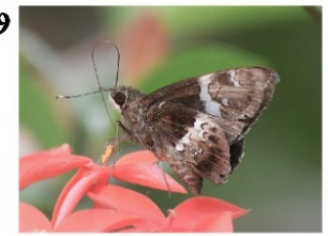

23

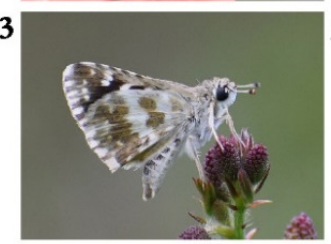

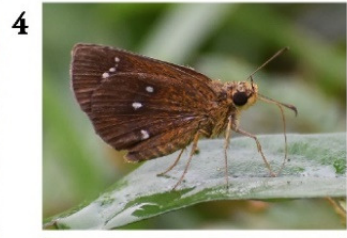

8
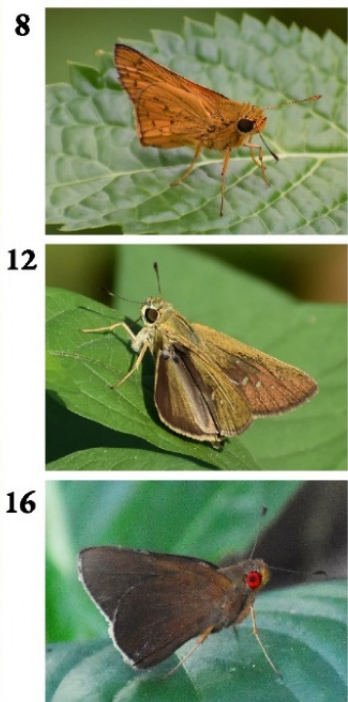

20

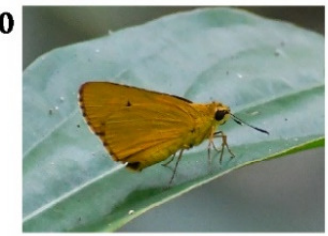

24

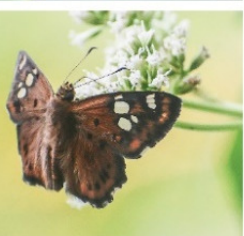

Figure 5. Photographs of butterfly species belonging to the family Hesperiidae recorded in this study: (1) Hasora chromus; (2) Hasora vitta; (3) Ampittia dioscorides; (4) Iambrix salsala; (5) Taractrocera maevius; (6) Matapa aria; (7) Parnara ganga; (8) Telicota aneilla; (9) Udaspes folus; (10) Suastus gremius; (11) Pelopidas subochracea; (12) Borbo bevani; (13) Pelopidas agna; (14) Telicota colon; (15) Cephrenes acalle, (16) Matapa purpurascens; (17) Borbo cinnara; (18) Pelopidas mathias; (19) Hyarotis adrastus; (20) Cupitha purreea; (21) Sarangesa dasahara; (22) Caprona ransonnetti; (23) Spialia galba; (24) Coladenia indrani; (25) Tagiades litigiosa 

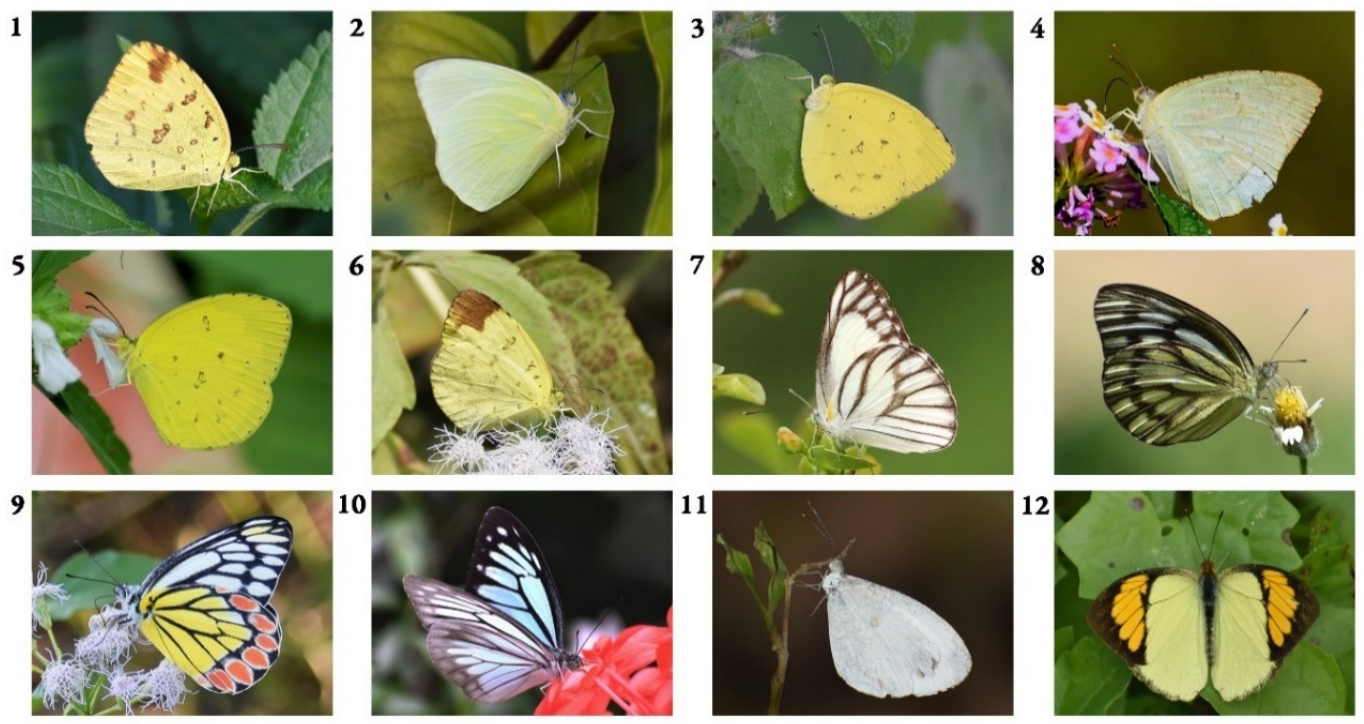

Figure 6. Photographs of butterfly species belonging to the family Pieridae recorded in this study: (1) Eurema simulatrix; (2) Catopsilia pomona; (3) Eurema hecabe; (4) Catopsilia pyranthe; (5) Eurema andersonii; (6) Eurema blanda; (7) Appias olferna; (8) Cepora nerissa; (9) Delias eucharis; (10) Pareronia hippia; (11) Leptosia nina; (12) Ixias pyrene 

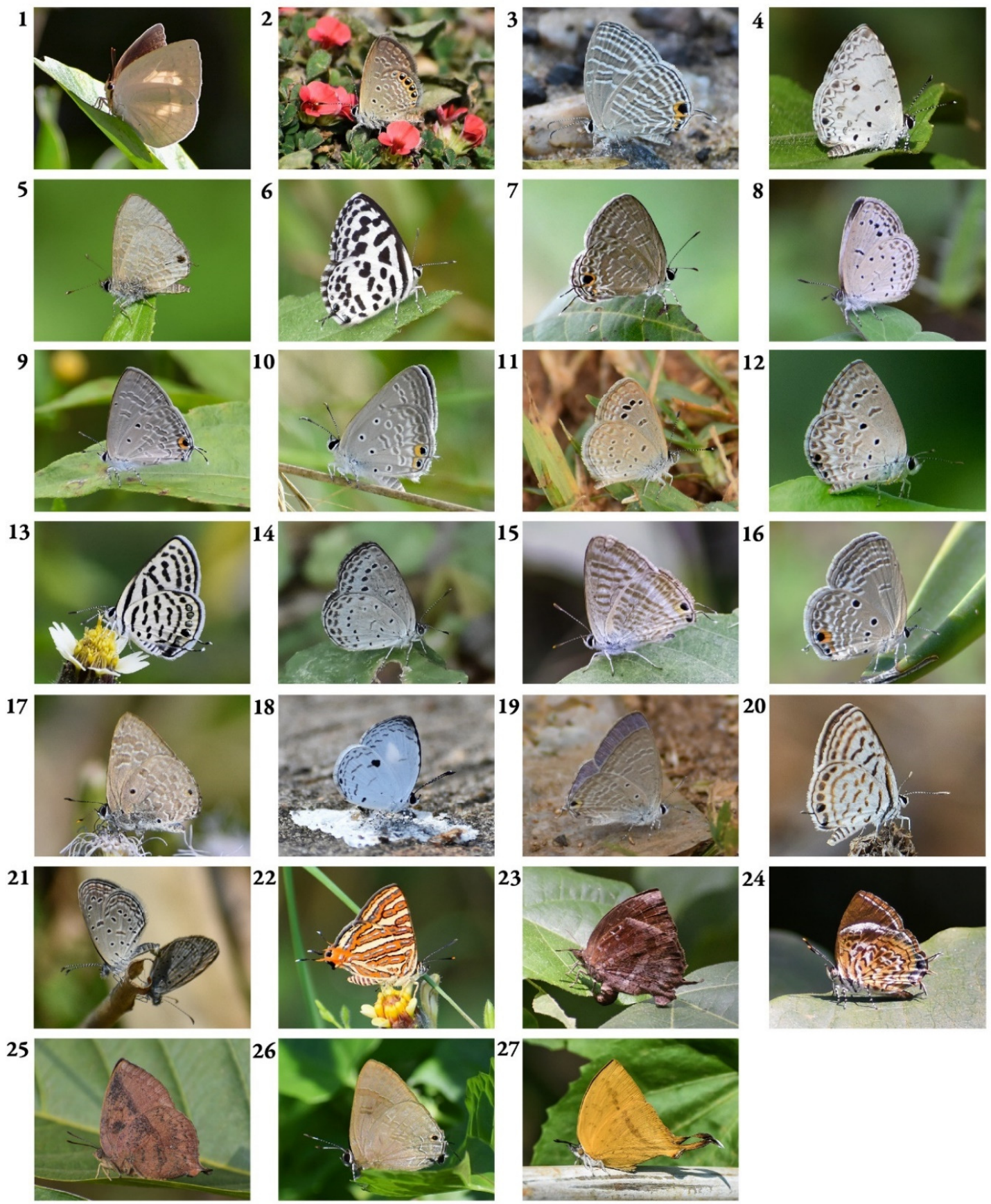

Figure 7. Photographs of butterfly species belonging to the family Lycaenidae recorded in this study: (1) Curetis thetis; (2) Freyeria putli; (3) Jamides celeno; (4) Acytolepis puspa; (5) Prosotas nora; (6) Castalius rosimon; (7) Jamides bochus; (8) Zizeeria karsandra; (9) Catochrysops strabo; (10) Euchrysops cnejus; (11) Zizina otis; (12) Chilades lajus; (13) Tarucus balkanica; (14) Pseudozizeeria maha; (15) Lampides boeticus; (16) Luthrodes pandava; (17) Anthene lycaenina; (18) Neopithecops zalmora; (19) Catochrysops panormus; (20) Tarucus nara; (21) Zizula hylax; (22) Spindasis vulcanus; (23) Mahathala ameria; (24) Rathinda amor; (25) Amblypodia anita; (26) Rapala manea; (27) Loxura atymnus 

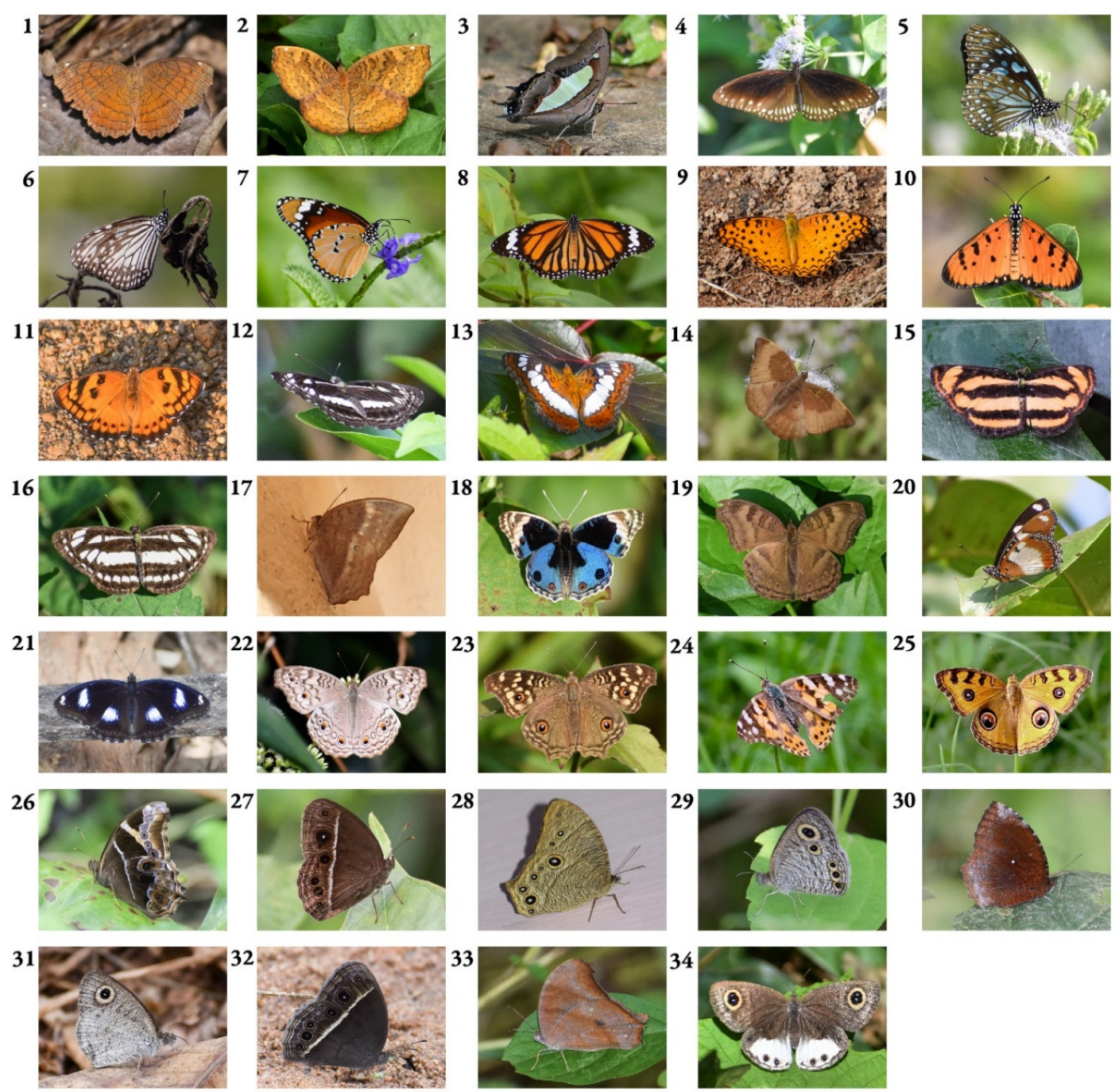

Figure 8. Photographs of butterfly species belonging to the family Nymphalidae recorded in this study: (1) Ariadne ariadne, (2) Ariadne merione; (3) Polyura athamas, (4) Euploea core, (5) Tirumala limniace, (6) Parantica aglea; (7) Danaus chrysippus; (8) Danaus genutia; (9) Phalanta phalantha; (10) Acraea terpsicore, (11) Symphaedra nais; (12) Neptis jumbah; (13) Moduza procris; (14) Euthalia aconthea; (15) Pantoporia hordonia; (16) Neptis hylas; (17) Discophora sondaica; (18) Junonia orithya; (19) Junonia iphita; (20) Hypolimnas misippus; (21) Hypolimnas bolina; (22) Junonia atlites; (23) Junonia lemonias; (24) Vanessa cardui; (25) Junonia almana; (26) Lethe europa; (27) Mycalesis perseus; (28) Melanitis leda; (29) Ypthima huebneri; (30) Elymnias hypermnestra; (31) Ypthima asterope; (32) Mycalesis mineus; (33) Melanitis phedima; (34) Ypthima ceylonica 

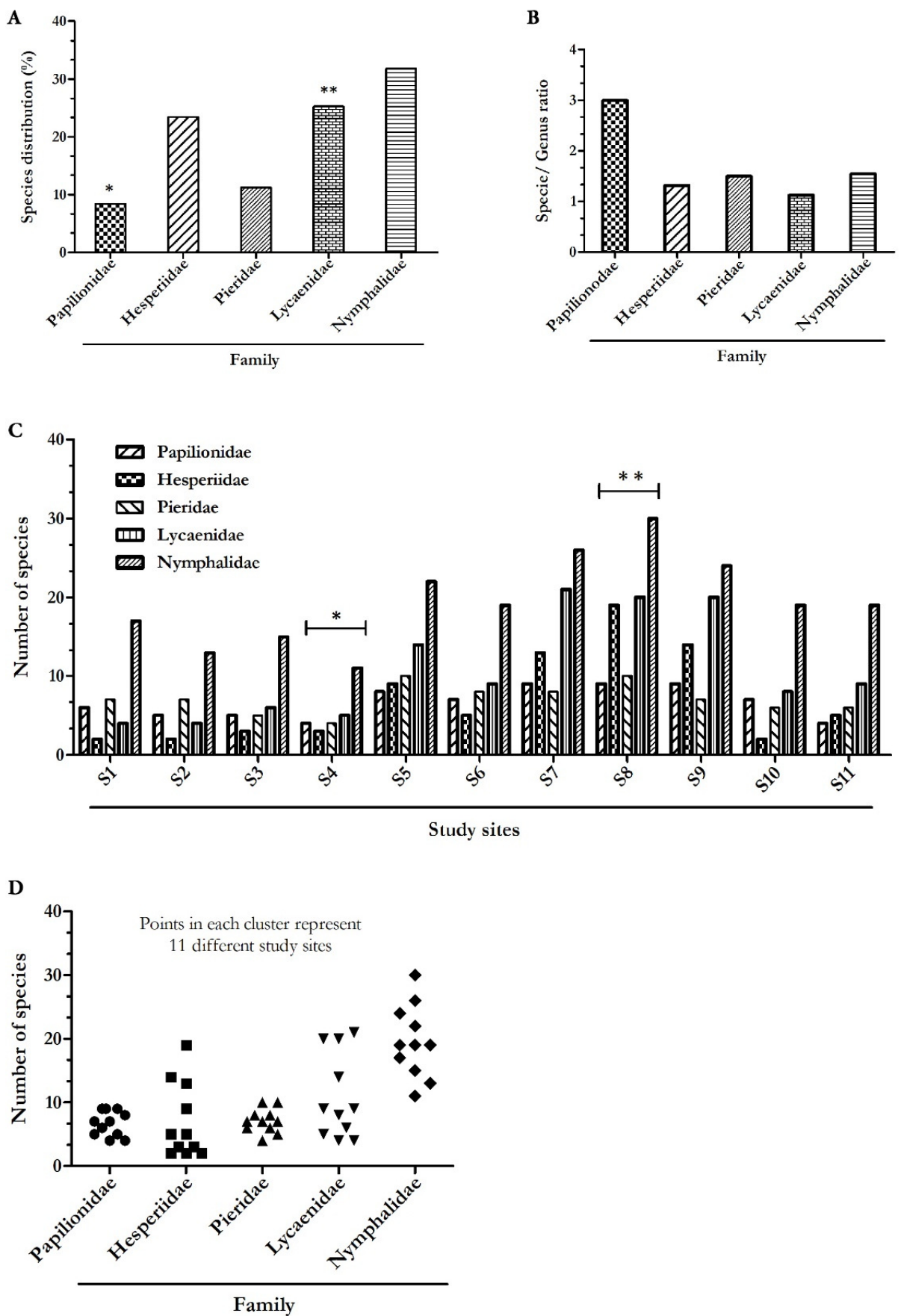

Figure 9. Butterfly species distribution reported in 11 different study sites: (A) Family wise distribution (\%) of species; (B) Species to genus ratio (S/G) observed in this study; (C) Number of species distributed in each study site; (D) Cluster graph showing family wise distribution range of species number in 11 different study sites

S, Study sites; ${ }^{*}$, Lowest species distribution; ${ }^{* *}$, Highest species distribution 
Table 4. Species to genus ratio for the recorded species in this study

\begin{tabular}{|l|c|c|c|c|}
\hline Sl. no & Family name & No. of Genus (G) & No. of Species (S) & S/G \\
\hline 1 & Papilionidae & 3 & 9 & 3.000 \\
\hline 2 & Hesperiidae & 19 & 25 & 1.316 \\
\hline 3 & Pieridae & 8 & 12 & 1.500 \\
\hline 4 & Lycaenidae & 24 & 27 & 1.125 \\
\hline 5 & Nymphalidae & 22 & 34 & 1.545 \\
\hline
\end{tabular}

Table 5. Sørensen's similarity index for butterfly species recorded in the study sites

\begin{tabular}{|l|c|c|c|c|c|c|c|c|c|c|c|}
\hline $\begin{array}{c}\text { Study } \\
\text { site }\end{array}$ & S1 & S2 & S3 & S4 & S5 & S6 & S7 & S8 & S9 & S10 & S11 \\
\hline S1 & & $\mathbf{0 . 8 9 6 ^ { * }}$ & 0.857 & 0.730 & 0.687 & 0.762 & 0.602 & 0.565 & 0.618 & 0.718 & 0.734 \\
\hline S2 & $\mathbf{0 . 8 9 6 ^ { * }}$ & & 0.862 & 0.793 & 0.617 & 0.734 & 0.556 & 0.521 & 0.571 & 0.712 & 0.703 \\
\hline S3 & 0.857 & 0.862 & & 0.852 & 0.660 & 0.756 & 0.595 & 0.541 & 0.593 & 0.684 & 0.753 \\
\hline S4 & 0.730 & 0.793 & 0.852 & & 0.578 & 0.693 & 0.500 & $\mathbf{0 . 4 7 0} \wedge$ & 0.535 & 0.580 & 0.657 \\
\hline S5 & 0.687 & 0.617 & 0.660 & 0.578 & & 0.775 & 0.743 & 0.689 & 0.745 & 0.667 & 0.623 \\
\hline S6 & 0.762 & 0.734 & 0.756 & 0.693 & 0.775 & & 0.688 & 0.618 & 0.689 & 0.689 & 0.681 \\
\hline S7 & 0.602 & 0.556 & 0.595 & 0.500 & 0.743 & 0.688 & & 0.824 & 0.848 & 0.639 & 0.650 \\
\hline S8 & 0.565 & 0.521 & 0.541 & $\mathbf{0 . 4 7 0} \wedge$ & 0.689 & 0.618 & 0.824 & & 0.852 & 0.600 & 0.611 \\
\hline S9 & 0.618 & 0.571 & 0.593 & 0.535 & 0.745 & 0.689 & 0.848 & 0.852 & & 0.621 & 0.650 \\
\hline S10 & 0.718 & 0.712 & 0.684 & 0.580 & 0.667 & 0.689 & 0.639 & 0.600 & 0.621 & & 0.588 \\
\hline S11 & 0.734 & 0.703 & 0.753 & 0.657 & 0.623 & 0.681 & 0.650 & 0.611 & 0.650 & 0.588 & \\
\hline
\end{tabular}

Similarity index value marked $\left({ }^{*}\right)$ is highest and marked $\left({ }^{\wedge}\right)$ is the lowest. S1-S11, study sites.

Table 6. Results of one-way ANOVA based on the site wise and family wise distribution of species number

\begin{tabular}{|c|c|c|c|c|c|c|c|c|}
\hline 1 & $\begin{array}{c}\text { Butterfly species among } \\
\text { different study sites }\end{array}$ & $\begin{array}{l}\text { Source of } \\
\text { variation }\end{array}$ & SS & df & MS & F stat & P-value & F crit \\
\hline & & Between groups & 872.33 & 10 & 87.23 & 2.34 & $0.026^{*}$ & 2.05 \\
\hline & & Within groups & 1641.6 & 44 & 37.31 & & & \\
\hline & & Total & 2513.9 & 54 & & & & \\
\hline \multirow[t]{4}{*}{2} & \multirow[t]{4}{*}{$\begin{array}{l}\text { Butterfly species in } \\
\text { different families }\end{array}$} & $\begin{array}{l}\text { Source of } \\
\text { variation }\end{array}$ & SS & df & MS & F stat & P-value & F crit \\
\hline & & Between groups & 1324.8 & 4 & 331.21 & 13.92 & $<0.0001^{* *}$ & 2.55 \\
\hline & & Within groups & 1189.1 & 50 & 23.78 & & & \\
\hline & & Total & 2513.9 & 54 & & & & \\
\hline
\end{tabular}

ANOVA, analysis of variance; SS, sum of squares; df, degrees of freedom; MS, mean squares; F stat, F statistic; P-value, probable value; $F$ crit, critical value of $F$ distribution. F stat values are significant at $\mathrm{p}<0.05$.

\section{Discussion}

Out of the total 107 species documented, three species (Papilio clytia, Pachliopta hector and Hypolimnas misippus) are listed in Schedule I, four species (Euchrysops cnejus, Lampides boeticus, Anthene lycaenina and Mahathala ameria) in Schedule II and two species (Hasora vitta and Hyarotis adrastus) in Schedule IV of WPA, 1972. The study shows highest S/G ratio for Papilionidae, probably due to their less genetic diversity as compared to other four families. Highest species diversity is observed in the study sites (S8, S7, and S9 respectively), which are mainly dominated by fragmented forest, open scrubs, and croplands having 
many host plants to sustain their lifecycle. Least number of butterfly species was recorded in the study sites S2 and S4 comprising urban habitat with continuous anthropogenic activities and less availability of host plants. 17 species are new additions to the existing butterfly data of Bhubaneswar. In this study, Painted Lady (Vanessa cardui) is reported only once in the month of April 2019, which could be a result of its well-known long-range migration (Stefanescu et al., 2016). The cluster is more scattered in case of Hesperiidae, Lycaenidae, and Nymphalidae as these families exhibit a greater number of species as compared to Papilionidae and Pieridae. Sørensen's similarity index showed the highest value between the study sites S1 and S2 indicating the highest species overlap, perhaps because they are in close proximity and share a similar type of habitat. Least Sørensen's similarity index has been recorded between the sites S4 and S8 which might be due to the presence of contrasting habitats. One-way ANOVA data suggest there is a significant variation between the butterfly species of different families within the study range. Moreover, a significant variation is observed between the butterfly species among the study sites. The ANOVA results show that the $\mathrm{P}<0.05$ and the value of $\mathrm{F}$ statistic is greater than the critical value of $\mathrm{F}$ distribution, thereby rejecting the null hypothesis. Butterflies are an integral part of our ecology and play an important role in maintaining the ecological balance. The present documentation provides a reference point and opens new ways of analytical research scopes. Researchers would find better sustainable approaches towards global conservation by understanding the biology of local populations and their dynamics pertaining to rapidly urbanizing geographical ranges. Studying species diversity has become more important in today's world as it serves as a checkpoint for awareness and understanding of interspecific and intraspecific interactions.

\section{Conclusions}

The study range supports a rich diversity of butterflies with a wide variety of plants which provide them an ideal breeding habitat. An attempt is made in this study to show the importance of a local area as a model geographical region with diverse habitats, suggesting the importance of local population in long term biodiversity studies and conservation. Therefore, it is imperative to understand the relative dependence of the butterfly species on their habitat. Considering such correlations would help in putting a check on the decreasing number of butterflies due to rapid urbanization and habitat destruction. Further studies on local butterflies of this region, covering various other aspects would undoubtedly contribute a lot towards solving the global issue of conservation of nature and its depleting species.

\section{Authors' Contributions}

The manuscript is conceptualized, drafted and supervised by SKS. AS and NP helped equally in data curation and all the authors analysed the data. All the authors contributed equally for the field work while review and editing were done by SKS. All the authors read and approved the final manuscript.

Ethical approval (for researches involving animals or humans)

In this study none of the butterfly species were collected, euthanized or killed by any means. Thus, the images of butterflies represented in the figures are the result of live photography. An entomological net was occasionally used and the butterflies were released unharmed to their natural habitat as soon as they were photographed. 


\section{Acknowledgements}

The authors are thankful to the Principal, B.J.B. Autonomous College and H.O.D., Zoology, B.J.B. Autonomous College, Bhubaneswar, Odisha, India for necessary permissions to perform the initial observations. The authors are grateful to B. Swarup K. Subudhi for his support during field trip and Lohit S. Singh for sharing the photograph of Painted Lady butterfly. Authors are also thankful to Yagnya P. Samal, Mitasha Samal, Swayamsiddha Swain and Shaswati S. Samal for giving constant encouragement. This research received no specific grant from any funding agency in the public, commercial, or not-for-profit sectors.

\section{Conflict of Interests}

The authors declare that there are no conflicts of interest related to this article.

\section{References}

Aduse-Poku K, Brattstrom O, Kodandaramaiah U, Lees DC, Brakefield PM, Wahlberg N (2015). Systematics and historical biogeography of the old-world butterfly subtribe Mycalesina (Lepidoptera: Nymphalidae: Satyrinae). BMC Evolutionary Biology 15(1):15-167. https://doi.org/10.1186/s12862-015-0449-3

Bingham CT (1905). The Fauna of British India, including Ceylon and Burma: Butterflies. Vol. I. Taylor and Francis, London.

Bingham CT (1907). The Fauna of British India, including Ceylon and Burma: Butterflies. Vol. II. Taylor and Francis, London.

Boruah B, Das GN, Payra A, Gogoi MJ, Dash SK, Tamuly T, ... Rout SD (2018). Assessment of Butterfly (Lepidoptera, Rhopalocera) Diversity in Manchabandha and Budhikhamari Reserve Forest, Mayurbhanj, Odisha, India. Asian Journal of Conservation Biology 7(1):51-65. http://www.ajcb.in/archive_july_18.php

Butterflies of India (2021). Indian foundation for butterflies. National Centre for Biological Sciences. Titli Trust, India. Retrieved 2021 September 04 from https://www.ifoundbutterflies.org/

Christopher AO (2020). Comparative analyses of diversity and similarity indices of west bank forest and block a forest of the International Institute of Tropical Agriculture (IITA) Ibadan, Oyo State, Nigeria. International Journal of Forestry Research 2020:4865845. https://doi.org/10.1155/2020/4865845

Cotton A, Fric Z, Gupta I, Gasse P, Inayoshi Y, Khoon K, ...Smetacek P (2015). A synoptic catalogue of the butterflies of India. Indinov Publishing, New Delhi, Butterfly Research Centre, Bhimtal, India.

Fernandez-Chacon A, Stefanescu C, Genovart M, Nichols JD, Hines JE, Paramo F, ...Oro D (2014). Determinants of extinction-colonization dynamics in Mediterranean butterflies: the role of landscape, climate and local habitat features. Journal of Animal Ecology 83(1):276-285. https://doi.org/10.1111/1365-2656.12118

Forest \& Environment Department (2020). Climatological Data of Orissa 2020. ENVIS Centre of Odisha's State of Environment. Ministry of Environment, Forests \& Climate Change, Forest \& Environment Department, Orissa, Govt of India. Retrived 2021 September 04 from http://www.orienvis.nic.in/indexl.aspx?lid=24\&mid=1\&langid=1\&linkid $=22$

Gogoi PP, Vinoj V, Swain D, Roberts G, Dash J, Tripathy S (2019). Land use and land cover change effect on surface temperature over Eastern India. Scientific Reports 9:8859. https://doi.org/10.1038/s41598-019-45213-z

Horsfield T, Moore F (1857). A Catalogue of the Lepidopterous Insects in the Museum of the Hon. East-India Company: Vol. I. WM. H. Allen and Co., London.

India Meteorological Department (2018). Annual report 2018. Information Science \& Knowledge Resource. India Meteorological Department, New Delhi, Ministry of Earth Sciences, Govt. of India. Retrieved 2021 September 04 from https://metnet.imd.gov.in/imdnews/ar2018.pdf

Karmakar T, Nitin R, Sarkar V, Baidya S, Mazumder S, Chandrasekharan V, ... Veino J (2018). Early stages and larval host plants of some northeastern Indian butterflies. Journal of Threatened Taxa 10(6):11780-11799. http://doi.org/10.11609/jott.3169.10.6.11780-11799 
Kawahara AY, Breinholt JW (2014). Phylogenomics provides strong evidence for relationships of butterflies and moths. Proceedings of Royal Society B: Biological Sciences 281(1788):20140970. https://doi.org/10.1098/rspb.2014.0970

Kehimkar I (2016). Butterflies of India (1st ed). Bombay Natural History Society. Mumbai, India.

Kim TK (2017). Understanding one-way ANOVA using conceptual figures. Korean Journal of Anesthesiology 70(1):2226. https://doi.org/10.4097/kjae.2017.70.1.22

Klein AM, Vaissiere BE, Cane JH, Steffan-Dewenter I, Cunningham SA, Kremen C, Tscharntke T (2007). Importance of pollinators in changing landscapes for world crops. Proceedings of the Royal Society B: Biological Sciences 274(1608):303-313. https://doi.org/10.1098/rspb.2006.3721

Kozak KM, Wahlberg N, Neild AF, Dasmahapatra KK, Mallet J, Jiggins CD (2015). Multilocus species trees show the recent adaptive radiation of the mimetic heliconius butterflies. Society of Systematic Biologists 64(3):505-524. https://doi.org/10.1093/sysbio/syv007

Kronforst MR, Papa R (2015). The functional basis of wing patterning in Heliconius butterflies: the molecules behind mimicry. Genetics 200(1):1-19. https://doi.org/10.1534/genetics.114.172387

Kunte K (2000). India, a Lifescape: butterflies of peninsular India. Universities Press (1st ed). A.P. India.

MacDonald ZG, Anderson ID, Acorn JH, Nielsen SE (2018). Decoupling habitat fragmentation from habitat loss: butterfly species mobility obscures fragmentation effects in a naturally fragmented landscape of lake islands. Oecologia 186(1):11-27. https://doi.org/10.1007/s00442-017-4005-2

Mandal D, Nandi D (1983). On Collection of Papilionidae from Orissa, India. Records of the Zoological Survey of India 81(1/2):355-368. http://recordsofzsi.com/index.php/zsoi/article/view/161275

Manesi Z, Van Lange PA, Pollet TV (2015). Butterfly Eyespots: Their Potential Influence on Aesthetic Preferences and Conservation Attitudes. PLoS One 10(11):e0141433. https://doi.org/10.1371/journal.pone.0141433

Marshall GFL, Nicéville LD (1882). The butterflies of India, Burmah and Ceylon. The Calcutta Central Press Co., Ld., Calcutta.

Mishra S, Mahapatra PK, Sinha S, Nayak HP, Mishra AK, Nair MV, ...Panda S (2010). Butterfly diversity of Nandankanan Wildlife Sanctuary, Orissa, India. E-planet 8(1):31-37. http://eplanet.co.in/images/Publication/EPLANE8.1.pdf

Mohapatra RK, Mishra AK, Mishra S, Parida SP (2013). A prelimnary assessment of Butterfly diversity in Utkal University Campus, Odisha. Zoo's 28(9):28-31. https://www.zoosprint.zooreach.org/index.php/zp/article/view/1282/1180

Nallu S, Hill JA, Don K, Sahagun C, Zhang W, Meslin C, ...Kronforst MR (2018). The molecular genetic basis of herbivory between butterflies and their host plants. Nature Ecology \& Evolution 2:1418-1427. https://doi.org/10.1038/s41559-018-0629-9

Nitin R, Balakrishnan V, Churi PV, Kalesh S, Prakash S, Kunte K (2018). Larval host plants of the butterflies of the Western Ghats, India. Journal of Threatened Taxa 10(4):11495-11550. http://doi.org/10.11609/jott.3104.10.4.11495-11550

Payra A, Dash SK, Das UP, Palei HS, Mishra AK (2019). Butterflies of Athgarh Forest Division, Odisha, Eastern India, with notes on some significant records. Acta Biologica Sibirica 5(3):188-198. https://doi.org/10.14258/abs.v5.i3.6593

Pellet J, Bried JT, Parietti D, Gander A, Heer PO, Cherix D, Arlettaz R (2012). Monitoring butterfly abundance: beyond Pollard walks. PLoS One 7(7):e41396-e41396. https://doi.org/10.1371/journal.pone.0041396

Pollard E, Yates TJ (1992). The extinction and foundation of local butterfly populations in relation to population variability and other factors. Ecological Entomology 17(3):249-254. https://doi.org/10.1111/j.13652311.1992.tb01055.x

Priyamvada, Mohapatra AK (2016). A preliminary study on diversity of butterflies (Lepidoptera: Macrolepidoptera) in Regional Institute of Education campus, Bhubaneswar, Odisha, India. Journal of Entomology and Zoology Studies 4(2):489-496.

Reddy CS, Khuroo AA, Krishna PH, Saranya KRL, Jha CS, Dadhwal VK (2014). Threat evaluation for biodiversity conservation of forest ecosystems using geospatial techniques: A case study of Odisha, India. Ecological Engineering 69:287-303. https://doi.org/10.1016/j.ecoleng.2014.05.006

Roy A, Devi BSS, Debnath B, Mutrthy MRS (2010). Geospatial Modelling for Identification of Potential Ecological Corridors in Orissa. Journal of the Indian Society of Remote Sensing 38(3):387-399. https://doi.org/10.1007/s12524-010-0042-6 
Royer RA, Austin JE, Newton WE (1998). Checklist and "Pollard Walk" Butterfly Survey Methods on Public Lands. The American Midland Naturalist 140(2):358-371. https://doi.org/10.1674/00030031(1998)140[0358:CAPWBS]2.0.CO;2

Ruszczyk A, Silva CF (1997). Butterflies select microhabitats on building walls. Landscape and Urban Planning 38(1/2):119-127. https://doi.org/10.1016/S0169-2046(97)00039-X

Salz A, Fartmann T (2017). Larval habitat preferences of a threatened butterfly species in heavy-metal grasslands. Journal of Insect Conservation 21:129-136. https://doi.org/10.1007/s10841-017-9961-7

Scheduled Insect species (2014). ENVIS Centre on Wildlife \& Protected Areas. Retrieved 2021 September 04 from http://wiienvis.nic.in/Database/ScheduleSpeciesDatabase_7969.aspx

Singh A, Mohanty LM, Tripathy A, Pradhan S (2020). Study of butterfly diversity in agronomy field, OUAT, Bhubaneswar, Odisha, India. Journal of Entomology and Zoology Studies 8(1):1028-1034.

Smetacek P (2018). A naturalist's guide to the butterflies of India, Pakistan, Nepal, Bhutan, Bangladesh and Sri Lanka (1st ed). Prakash Books India Pvt. Ltd., New Delhi, India.

Stefanescu C, Soto DX, Talavera G, Vila R, Hobson KA (2016). Long-distance autumn migration across the Sahara by painted lady butterflies: exploiting resource pulses in the tropical savannah. Biology Letters 12(10):20160561. https://doi.org/10.1098/rsbl.2016.0561

Swain D, Roberts GJ, Dash J, Vinoj V, Lekshmi K, Tripathy S (2016). Impact of rapid urbanization on the microclimate of Indian cities: a case study for the city of Bhubaneswar. Proceedings Volume 9877, Land Surface and Cryosphere Remote Sensing III. SPIE Asia-Pacific Remote Sensing, New Delhi, India pp 98772X-7. https://doi.org/10.1117/12.2228111

Syaripuddin K, Sing K-W, Wilson J-J (2015). Comparison of Butterflies, Bats and Beetles as Bioindicators Based on Four Key Criteria and DNA Barcodes. Tropical Conservation Science 8(1):138-149. https://doi.org/10.1177/194008291500800112

Talbot G (1939). The Fauna of British India, including Ceylon and Burma: Butterflies. Vol. I. Taylor and Francis, London.

Talbot G (1947). The Fauna of British of India, including Pakistan, Ceylon and Burma: Butterflies. Vol. II. Taylor and Francis, London.

Thomas CD, Thomas JA, Warren MS (1992). Distributions of occupied and vacant butterfly habitats in fragmented landscapes. Oecologia 92(4):563-567. https://doi.org/10.1007/BF00317850

Tiple AD, Khurad AM, Dennis RLH (2011). Butterfly larval host plant use in a tropical urban context: life history associations, herbivory, and landscape factors. Journal of Insect Science 11(1):65. https://doi.org/10.1673/031.011.6501

van Bergen E, Osbaldeston D, Kodandaramaiah U, Brattstrom O, Aduse-Poku K, Brakefield PM (2017). Conserved patterns of integrated developmental plasticity in a group of polyphenic tropical butterflies. BMC Evolutionary Biology 17:59. https://doi.org/10.1186/s12862-017-0907-1

Van Nieukerken EJ, Kaila L, Kitching IJ, Kristensen NP, Lees DC, Minet J, ... Zwick A (2011). Order Lepidoptera. In: Zhang ZQ (Ed). Animal Biodiversity: An outline of higher-level classification and survey of taxonomic richness. Zootaxa 3148:212-221. https://doi.org/10.11646/zootaxa.3148.1.41

Verspagen N, Ikonen S, Saastamoinen M, van Bergen E (2020). Multidimensional plasticity in the Glanville fritillary butterfly: larval performance is temperature, host and family specific. Proceedings of the Royal Society B: Biological Sciences 287(1941):20202577. https://doi.org/10.1098/rspb.2020.2577

Wang Wei J, Lee BPY-H, Bing Wen L (2016). Citizen Science and the Urban Ecology of Birds and Butterflies - A Systematic Review. PLoS One 11(6):e0156425. https://doi.org/10.1371/journal.pone.0156425

Wood B, Gillman MP (1998). The effects of disturbance on forest butterflies using two methods of sampling in Trinidad. Biodiversity \& Conservation 7:597-616. https://doi.org/10.1023/A:1008800317279 
OPEN ACCESS

(c) (1)

The journal offers free, immediate, and unrestricted access to peer-reviewed research and scholarly work. Users are allowed to read, download, copy, distribute, print, search, or link to the full texts of the articles, or use them for any other lawful purpose, without asking prior permission from the publisher or the author.

License - Articles published in Notulae Scientia Biologicae are Open-Access, distributed under the terms and conditions of the Creative Commons Attribution (CC BY 4.0) License.

(C) Articles by the authors; SHST, Cluj-Napoca, Romania. The journal allows the author(s) to hold the copyright/to retain publishing rights without restriction. 